EMPG-15-02

\title{
Stratified Fiber Bundles, Quinn Homology and Brane Stability of Hyperbolic Orbifolds
}

\author{
Andrey A. Bytsenko ${ }^{(a)}$ 月, Richard J. Szabo ${ }^{(b)}$ 2 and Anca Tureanu ${ }^{(c)}$ 3 \\ (a) Departamento de Física, Universidade Estadual de Londrina \\ Caixa Postal 6001, Londrina-Paraná, Brazil \\ (b) Department of Mathematics, Heriot-Watt University \\ Colin Maclaurin Building, Riccarton, Edinburgh EH14 4AS, UK \\ Maxwell Institute for Mathematical Sciences, Edinburgh, UK \\ The Higgs Centre for Theoretical Physics, Edinburgh, UK \\ (c) Department of Physics, University of Helsinki \\ P.O. Box 64, FI-00014 Helsinki, Finland
}

\begin{abstract}
We revisit the problem of stability of string vacua involving hyperbolic orbifolds using methods from homotopy theory and K-homology. We propose a definition of Type II string theory on such backgrounds that further carry stratified systems of fiber bundles, which generalise the more conventional orbifold and symmetric string backgrounds, together with a classification of wrapped branes by a suitable generalized homology theory. For spaces stratified fibered over hyperbolic orbifolds we use the algebraic K-theory of their fundamental groups and Quinn homology to derive criteria for brane stability in terms of an Atiyah-Hirzebruch type spectral sequence with its lift to K-homology. Stable D-branes in this setting carry stratified charges which induce new additive structures on the corresponding K-homology groups. We extend these considerations to backgrounds which support $H$-flux, where we use K-groups of twisted group algebras of the fundamental groups to analyse stability of locally symmetric spaces with K-amenable isometry groups, and derive stability conditions for branes wrapping the fibers of an Eilenberg-MacLane spectrum functor.
\end{abstract}

\footnotetext{
1aabyts@gmail.com

${ }^{2}$ R.J.Szabo@hw.ac.uk

3 anca.tureanu@helsinki.fi
} 


\section{Contents}

1 Introduction and summary 2

2 String orbifolds, stratified fiber bundles and branes 3

3 Quinn homology

4 Branes in spaces stratified fibered over orbifolds $\quad 10$

5 Inclusion of $H$-flux $\quad 16$

\section{Introduction and summary}

Backgrounds of string theory with group actions have a distinctive place in the landscape of string vacua, as they provide a procedure for generating new backgrounds from old ones. For example, just as strings can propagate on spaces, they can also propagate on orbifolds [1, 2, 3] which lead to many novel stringy effects, particularly in the description of D-branes. Moreover, all supergravity backgrounds which preserve more than half the supersymmetry are homogeneous [4], and symmetric spaces constitute a special class wherein the classification of these backgrounds can be substantially progressed.

In this paper we revisit the problem of supersymmetry of string theory backgrounds involving hyperbolic orbifolds; in fact we analyse the weaker problem of stability of these backgrounds. In Sect. 2 we review the significance of these string vacua and problems associated with their supersymmetry. Starting from features of string propagation in orbifolds and in symmetric spaces, we then consider modifications of these backgrounds wherein they are regarded as the bases of stratified systems of fiber bundles, and more specifically of spaces stratified fibered over hyperbolic orbifolds. We propose a definition of Type II string theory on these spaces and analyse their stability in terms of wrapped branes, which constitute novel extensions of string theory on orbifold and symmetric backgrounds. The existence of a stratification on a background $\mathcal{B}$ is of course neither novel nor exotic: If $\mathcal{B}$ is endowed with the structure of a simplicial complex, then the strata are just the open simplices. However, the more pertinent example to bear in mind throughout this paper is that of an orbifold, which is a disjoint union of strata with each stratum a connected component of points with the same isotropy group (up to conjugacy).

In this paper we shall introduce and emphasise new methods from homotopy theory for the classification of branes on these broader classes of singular backgrounds, which are techniques that have received considerably less attention in the physics literature compared to e.g. Ktheory. The basic idea is that by considering these backgrounds and their branes in stratified families we can probe their stability by endowing them with "local coefficient systems", and then classify them by a suitable homology theory on the category of spaces with stratified systems of fiber bundles. This is homology with twisted and stratified spectrum coefficients 
developed by Quinn [5]; we give a general overview of Quinn homology in Sect. [3] using the language of homology loop spectra, paying particular attention to an Atiyah-Hirzebruch type spectral sequence and its relation to singular or C̆ech homology. As we discuss in the following, Quinn homology for stratified systems of fiber bundles plays a role analogous to Bredon homology for orbifolds.

In Sect. 4 we discuss the classification of stable D-branes in spaces stratified fibered over hyperbolic orbifolds. We use a suitable Atiyah-Hirzebruch type spectral sequence and stratified systems of abelian groups over hyperbolic manifolds to derive rigorous stability criteria. As the initial homology charges are have coeffcients valued in stratified systems of groups, stable D-branes carry charges which induce new additive structures on K-homology. For backgrounds with strongly virtually negatively curved fundamental groups $\Lambda$, we give an explicit description of the homology groups in which the obstructions to D-brane stability lie in terms of the algebraic K-theory of $\Lambda$. We also find a broad class of novel examples of unstable backgrounds.

Finally, in Sect. 5 we extend our considerations to examples involving Type II D-branes in $H$ flux backgrounds, using their description in terms of KK-groups and the twisted K-homology of continuous trace $C^{*}$-algebras. In the case of locally symmetric spaces with K-amenable isometry groups, we provide an explicit analysis of stability conditions using K-groups of the twisted group $C^{*}$-algebra of the fundamental group of the background. In particular, when the locally symmetric background is an Eilenberg-MacLane space, we examine conditions under which it can be extended via a stratified system of abelian groups through a suitable Atiyah-Hirzebruch type spectral sequence as a non-trivial element of the homology groups; stability here is formulated in terms of filtration structured spectra associated to the fibers of the Eilenberg-MacLane spectrum functor.

\section{$2 \quad$ String orbifolds, stratified fiber bundles and branes}

Orbifolds. Let $\Lambda$ be a discrete group acting properly on a smooth manifold $X$ with finite stabilizers. A Type II string orbifold is then constructed by taking a worldsheet $\Sigma$, which is a compact oriented two-manifold, and a collection of smooth maps $\phi: \Sigma \rightarrow X$. To include twisted sectors into the string theory, we consider a larger space of fields which is a groupoid; the objects of the groupoid label the twisted sectors and are pairs $(P, \tilde{\phi})$ consisting of a principal $\Lambda$-bundle $P \rightarrow \Sigma$ with a $\Lambda$-equivariant map $\tilde{\phi}: P \rightarrow X$, while the morphisms $(P, \tilde{\phi}) \rightarrow\left(P^{\prime}, \tilde{\phi}^{\prime}\right)$ are given by isomorphisms $f: P \rightarrow P^{\prime}$ of principal $\Lambda$-bundles such that $\tilde{\phi}=\tilde{\phi}^{\prime} \circ f$. This defines the string theory on the global quotient spacetime $\mathcal{B}=[X / \Lambda]$, which can be presented as the action groupoid $\Lambda \times X \rightrightarrows X$ whose objects are the points $x \in X$ and whose morphisms $x \rightarrow y$ are the group elements $g \in \Lambda$ for which $g \cdot x=y$; the groupoid structure keeps track of the isotropy subgroups $\Lambda_{x}:=\{h \in \Lambda \mid h \cdot x=x\} \subset \Lambda$ for all $x \in X$, and a string field $\phi: \Sigma \rightarrow \mathcal{B}$ is given by a pair $(P, \tilde{\phi})$ as above. A geometric realization of this quotient is given by the Borel construction $|\mathcal{B}|:=\underline{E} \Lambda \times_{\Lambda} X$, where $\underline{E} \Lambda$ is a contractible space with a free $\Lambda$-action; if $X$ is contractible then the orbifold fundamental group is $\pi_{1}(\mathcal{B})=\pi_{1}(|\mathcal{B}|)=\Lambda$. For further details and background on orbifolds, see e.g. [6]. 
We can also admit as spacetimes more general orbifolds $\mathcal{B}$ which are not global quotients by discrete groups. Generally, orbifolds are presented as groupoids, so that points can have automorphisms $\Lambda_{x}$ as above, or alternatively as smooth real Deligne-Mumford stacks; see [7] for an introductory exposition of results that we shall use throughout this paper. Recall that a stack is an object in the 2-category of sheaves of groupoids on the category of smooth manifolds with respect to the usual Grothendieck topology given by open coverings. Any manifold $X$ is itself a stack which associates to each test manifold $T$ the (trivial) groupoid of smooth maps $X(T)=C^{\infty}(T, X)$. The global quotient stack $[X / \Lambda]$ associates to $T$ the groupoid $[X / \Lambda](T)$ of pairs $(P \rightarrow T, \tilde{\phi})$ as above; if $\Lambda$ acts freely with quotient manifold $X / \Lambda$, then there is a natural isomorphism of stacks $[X / \Lambda] \cong X / \Lambda$ or alternatively a Morita equivalence between the action groupoid $\Lambda \times X \rightrightarrows X$ and the unit groupoid $X / \Lambda \rightrightarrows X / \Lambda$. The maps between two stacks $\mathcal{B} \rightarrow \mathcal{B}^{\prime}$ form the groupoid $\operatorname{Hom}\left(\mathcal{B}, \mathcal{B}^{\prime}\right)$ of maps between sheaves of groupoids; the objects of $\operatorname{Hom}\left(\mathcal{B}, \mathcal{B}^{\prime}\right)$ are morphisms $f: \mathcal{B} \rightarrow \mathcal{B}^{\prime}$ of stacks and the morphisms of $\operatorname{Hom}\left(\mathcal{B}, \mathcal{B}^{\prime}\right)$ are 2-morphisms $f_{1} \Rightarrow f_{2}$ between morphisms $f_{1}, f_{2}: \mathcal{B} \rightarrow \mathcal{B}^{\prime}$. The Yoneda embedding then gives a Morita equivalence of groupoids $\mathcal{B}(T) \cong \operatorname{Hom}(T, \mathcal{B})$. The string fields can thus be regarded as maps $\phi: \Sigma \rightarrow \mathcal{B}$ of stacks, and the groupoid of all such maps includes twisted sectors of the orbifold string theory. If the stack $\mathcal{B}$ is presented as a groupoid $\mathcal{G}=\left(\mathcal{G}_{1} \rightrightarrows \mathcal{G}_{0}\right)$, then the collection of $n$-tuples of composable morphisms $\mathcal{G}_{n}=\left(\mathcal{G}_{1}\right)^{\times \mathcal{G}_{0}}{ }^{n}$ is a simplicial space with geometric realisation $|\mathcal{B}|=\coprod_{n} \mathcal{G}_{n} \times \Delta^{n} / \sim$, where $\Delta$ denotes the standard simplicial 2-category of ordered simplices and the equivalence relation $\sim$ identifies the face and degeneracy maps of $\mathcal{G}$ and $\Delta$; throughout $\Delta^{n}$ denotes the standard $n$-simplex. The set of isomorphism classes of objects of the groupoid is called the orbit space of the orbifold, or the coarse moduli space of the stack. For further details concerning stacks in relation to string orbifolds, see [2].

Other geometric data, such as metrics, connections and supergravity form fields, can also be defined in this setting. In particular, D-branes wrapping $\mathcal{B}$ carry gauge fields and require a notion of Chan-Paton bundles on stacks. A vector bundle on a stack $\mathcal{B}$ is a map of stacks from $\mathcal{B}$ to the stack of vector bundles whose evaluation on a test manifold $T$ is the groupoid of vector bundles over $T$ and isomorphisms. Alternatively, given a groupoid presentation s, t : $\mathcal{G}_{1} \rightrightarrows \mathcal{G}_{0}$ for $\mathcal{B}$, a vector bundle on $\mathcal{B}$ is a vector bundle $\mathcal{E} \rightarrow \mathcal{G}_{0}$ together with an isomorphism $\mathrm{s}^{*} \mathcal{E} \stackrel{\sim}{\rightarrow} \mathrm{t}^{*} \mathcal{E}$ of bundles over $\mathcal{G}_{1}$ which satisfies a cocycle condition over $\mathcal{G}_{2}=\mathcal{G}_{1} \times{ }_{\mathcal{G}_{0}} \mathcal{G}_{1}$. Finite rank vector bundles on a groupoid and their morphisms form a symmetric monoidal category; the Grothendieck group of this category computes the Ktheory of the stack $\mathcal{B}$.

Stratified fiber bundles. In this paper we will be concerned with a homotopy theoretic generalization of the class of string backgrounds given by orbifolds. For this, recall that a stratification of a space $\mathcal{B}$ is a locally finite decomposition $\mathcal{B}=\coprod_{i} \mathcal{B}_{i}$ into pairwise disjoint and locally closed submanifolds $\mathcal{B}_{i}$, called strata. The usual homotopy lifting property which defines a fiber bundle is generalized to the stratified setting as follows: A map $p: X \rightarrow \mathcal{B}$ between stratified spaces is called a stratified fiber bundle if, for any map $f: E \rightarrow X$, every stratum preserving homotopy $F: E \times \Delta^{1} \rightarrow \mathcal{B}$ from $\left.F\right|_{0}=p \circ f$ lifts to a stratified homotopy $\tilde{F}: E \times \Delta^{1} \rightarrow X$ from $\left.\tilde{F}\right|_{0}=f$ with $p \circ \tilde{F}=F$. See e.g. [8] for further details and properties.

The global quotient orbifolds describing string backgrounds fit into this framework in the 
following way. Suppose that $\Lambda$ is a finite group acting smoothly on a manifold $X$. For a subgroup $H \subseteq \Lambda$, let $X_{(H)}$ be the $\Lambda$-invariant submanifold of $X$ consisting of all points $x \in X$ whose isotropy subgroup $\Lambda_{x}$ is conjugate to $H$; then $X_{(\Lambda)}$ is the fixed point locus of the $\Lambda$-action, while $X_{(1)}$ is the set of points on which $\Lambda$ acts freely, i.e., the regular points which form a dense open subset of $\mathcal{B}=[X / \Lambda]$. The distinct sets $X_{(H)}$ depend only on the conjugacy class of $H \subseteq \Lambda$ and give the strata of the stabilizer stratification of $X$; the corresponding quotients $X_{(H)} / \Lambda$ give the orbit stratification of the orbit space $X / \Lambda$ with the factor topology. Then the orbit map $X \rightarrow X / \Lambda$ is a stratified fiber bundle of coarse moduli spaces underlying the map of stacks $X \rightarrow \mathcal{B}$ which defines a principal $\Lambda$-bundle over $\mathcal{B}$. A similar construction holds for stacks which are presentable as local quotient groupoids, i.e., as groupoids which admit a countable open cover $\left\{\mathcal{U}_{i}\right\}$ such that the restriction to each $\mathcal{U}_{i}$ is Morita equivalent to an action groupoid $\Lambda_{i} \times U_{i} \rightrightarrows U_{i}[9]$.

Symmetric spaces. An important generalization of this last construction of stratified fiber bundles is to the case where $\Lambda$ is replaced by a compact Lie group $\Gamma$. Then one can regard the covering projections $X \rightarrow X / \Gamma$ as a collection of fiber bundles by the property that each orbit map $X_{(H)} \rightarrow X_{(H)} / \Gamma$, for $H \subseteq \Gamma$, is a smooth fiber bundle with fibers $\Gamma / H$.

This extension pertains in particular to the class of homogeneous Type II string backgrounds given by (locally) symmetric spaces. For this, let $G$ be a connected semisimple Lie group without compact factors. Let $\Gamma \subset G$ be a maximal compact subgroup, and let $\Lambda \subset G$ be a cocompact torsion-free lattice. Then the contractible manifold $X=G / \Gamma$ carries a $G$ invariant Riemannian metric and is a non-compact symmetric space. The discrete group $\Lambda$ acts freely and properly on $X$ via $\lambda \cdot(g \Gamma):=(\lambda g) \Gamma$ for $\lambda \in \Lambda$ and $g \in G$, and the closed manifold $\mathcal{B}=\Lambda \backslash X$ is a compact locally symmetric space with the factor topology which is a model for the classifying stack $\underline{B} \Lambda=\underline{E} \Lambda / \Lambda \cong[\mathrm{pt} / \Lambda]$ of principal $\Lambda$-bundles with $X=\underline{E} \Lambda$ a model for the universal space for proper $\Lambda$-actions. If $\Lambda$ has torsion, then the $\Lambda$-action on $X$ need not be free since the isotropy subgroups are $\Lambda_{x}=\left(x^{-1} \Lambda x\right) \cap \Gamma$ for $x \in X$ (which are finite as they are discrete subgroups of the compact group $\Gamma$ ); in this instance $\mathcal{B}$ is a stratified space as above. Such backgrounds preserve more than half of the supersymmetry; backgrounds preserving a substantial amount of supersymmetry play a crucial role in string theory, particularly in gauge theory/gravity correspondences.

Stability of hyperbolic orbifolds. In the case that $G$ is a connected Lie group of real rank one and $\mathcal{B}=\Lambda \backslash X=\Lambda \backslash G / \Gamma$ is a compact locally symmetric Riemannian manifold of dimension $n$ with negative sectional curvature, the covering manifold $X$ is a real hyperbolic space $H^{n}$. We shall mostly focus on these spaces as they yield a class of backgrounds $\mathcal{B}$ which are potentially compatible with supersymmetry in our constructions to follow; in particular, as supergravity solutions they naturally arise as near-horizon geometries of branes.

The $n$-dimensional real hyperbolic space can be represented as the symmetric space $H^{n}=$ $G / \Gamma$, where $G=S O_{1}(n, 1)$ and $\Gamma=S O(n)$ is a maximal compact subgroup of $G$. They admit Killing spinors [10, 11, 12, 13], but they have infinite volume with respect to the Poincaré metric and hence do not support field configurations with normalizable zero modes. On the other hand, consistent field theories can be obtained by taking backgrounds $\mathcal{B}$ with the topology of coset spaces $H^{n} / \tilde{\Lambda}$, where $\tilde{\Lambda}$ is a discrete subgroup of $G$ acting isometrically on $H^{n}$. 
The question of whether such quotient spaces admit Killing spinors and preserve supersymmetry is addressed for some examples of finite volume hyperbolic spaces in [14]. Moreover, a version of the gauge theory/gravity correspondence states that string theory (or M-theory) compactified on spaces of the form $A d S_{d+1} \times\left(H^{n} / \tilde{\Lambda}\right) \times S^{k}$ defines a $d$-dimensional conformal field theory with $S O(k+1)$ global symmetry. We mention three such particular cases of supergravity solutions from [14] which will be pertinent later on. Firstly, the 11-dimensional supergravity solution of the form $A d S_{5} \times\left(H^{2} / \tilde{\Lambda}\right) \times S^{4}$ is dual to a $d=4$ non-supersymmetric conformal field theory with $S O(5)$ global symmetry group; it carries M5-brane charge and is expected to be related to the six-dimensional $(2,0)$ or $(1,0)$ superconformal field theories. Secondly, the 11-dimensional supergravity solution $A d S_{4} \times\left(H^{3} / \tilde{\Lambda}\right) \times S^{4}$ has M5-brane charge, and thus its dual field theory is a $d=3$ conformal field theory associated with the six-dimensional $(2,0)$ superconformal theory with an internal global symmetry group $S O(5)$. Thirdly, the Type IIB supergravity solution $A d S_{3} \times\left(H^{2} / \tilde{\Lambda}\right) \times S^{5}$ has D3-brane charge and it should be dual to some non-supersymmetric $d=2$ conformal field theory related to $\mathcal{N}=4$ supersymmetric Yang-Mills theory in four dimensions; these models fall into the class of regular string compactifications (modulo possible orbifold points) which have small $\alpha^{\prime}$ string corrections and are holographically dual to $\mathcal{N}=0$ conformal field theories in four dimensions [14, 15, 16].

Various string backgrounds can be presented by means of direct products of spaces containing real hyperbolic space forms as factors; for example the space forms $A d S_{3} \times H^{2} \times H^{2} \times S^{4}$ and $A d S_{2} \times H^{2} \times H^{3} \times S^{4}$ appear as solutions of 11-dimensional supergravity. However, it is easy to show that any connected locally Riemannian product $M=H^{k} \times H^{l}$ cannot leave any unbroken supersymmetry. For this, suppose that $M$ admits a Killing spinor $\psi \neq 0$ with Killing number $\mu \neq 0$; then $M$ is locally irreducible [17. Let $\xi$ and $\eta$ be (pullbacks to $M$ of) vector fields on $H^{k}$ and $H^{l}$ respectively; then $\xi \cdot \eta=0$ and $\eta \cdot \xi=0$. The Killing spinor equations at each point of $M$ then read as $\nabla_{\xi} \psi=\mu \xi \cdot \psi$ and similarly for $\nabla_{\eta}$, where $4 \mu^{2}=(n(n-1))^{-1} R$ and $R$ is the scalar curvature (here $n=k+l$ ). Thus we have

$$
R(\xi, \eta) \psi=\left(\nabla_{\xi} \nabla_{\eta}-\nabla_{\eta} \nabla_{\xi}-\nabla_{[\xi, \eta]}\right) \psi=\mu^{2}[\eta, \xi] \cdot \psi=0
$$

and since $R \neq 0$ it follows that $\psi=0$, which is a contradiction. Other compactifications can be obtained by replacing hyperbolic space forms $H^{n}$ by any Einstein space $M$ of the same negative curvature and with Killing spinors; in fact, if $M$ is a connected Riemannian Spin manifold admitting a non-trivial Killing spinor with non-zero Killing number, then $M$ is an Einstein space [17]. In particular, one can consider $\left(H^{k} \times H^{l}\right) / \tilde{\Lambda}$ and ask if the orbifold by the discrete group $\tilde{\Lambda}$ restores supersymmetry. But even if non-supersymmetric, these solutions are regular everywhere (being a direct product of Einstein spaces) and $\alpha^{\prime}$ corrections can be made small for sufficiently large radius of the compact parts. Hence like the Type IIB supergravity backgrounds $A d S_{3} \times H^{2} \times S^{5}$ and $A d S_{2} \times H^{3} \times S^{5}$ of [14] with D3brane charge and constant dilaton, these spaces could still constitute consistent backgrounds for string compactification, and in particular for the construction of conformal field theories. In general a definitive statement about the stability of supergravity solutions requires study of the spectral properties of certain operators, which is largely undeveloped as of yet.

Spaces stratified fibered over hyperbolic orbifolds. To address these stability issues, 
we add more structure to this construction: to detect possible instabilities we consider the background $\mathcal{B}$ in suitable families which "probe" the structure of the orbifold. Following [5, Sect. 8], let $\mathcal{B}=\mathcal{B}^{n} \supseteq \mathcal{B}^{n-1} \supseteq \cdots \supseteq \mathcal{B}^{0}$ be a closed finite filtration of the space $\mathcal{B}$; then one obtains a stratification of $\mathcal{B}$ by setting $\mathcal{B}_{i}:=\mathcal{B}^{i}-\mathcal{B}^{i-1}$ for $i=0,1, \ldots, n$, where $\mathcal{B}^{-1}:=\varnothing$. We will usually use a skeletal filtration whereby $\mathcal{B}^{i}$ is the $i$-skeleton of a $\mathrm{CW}$-decomposition of $\mathcal{B}$; then the strata $\mathcal{B}_{i}$ are the open $i$-simplices. A map $p: X \rightarrow \mathcal{B}$ is called a stratified system of fiber bundles on $\mathcal{B}$ if the restriction to each stratum $p \mid: p^{-1}\left(\mathcal{B}_{i}\right) \rightarrow \mathcal{B}_{i}$ is an ordinary fiber bundle, and each term in the filtration $\mathcal{B}^{i}$ is a $p$-neighborhood deformation retract subset of $\mathcal{B}$, i.e., there exists a neighborhood of $\mathcal{B}^{i}$ in $\mathcal{B}$, with a continuous deformation into $\mathcal{B}$ that preserves all strata and is covered by a deformation of its fibers in $X$. In the case of string orbifolds, with the discrete group $\Lambda$ acting cellularly on $X$, the projection of the geometric realisation $|\mathcal{B}|=\underline{E} \Lambda \times_{\Lambda} X$ to the second factor defines a stratified system of fiber bundles $p:|\mathcal{B}| \rightarrow X / \Lambda$ [18]; in this case there is a homotopy equivalence $p^{-1}(\sigma) \cong \underline{B} \Lambda_{\sigma}$ for any simplex $\sigma$ of $\mathcal{B}=[X / \Lambda]$.

Now let $M$ be a closed connected Riemannian manifold with strictly negative sectional curvatures and let $\left\{\mathcal{X}^{j}\right\}_{j=1}^{\infty}, \mathcal{X}^{1} \subset \mathcal{X}^{2} \subset \cdots$ be a sequence of connected compact smooth manifolds. Let $\tilde{\Lambda}$ be a finite group acting on $M$ freely via isometries and on each $\mathcal{X}^{j}$ via smooth maps; the action of $\tilde{\Lambda}$ on $\mathcal{X}^{j}$ need not be free. Assume moreover that the smooth embedding $\mathcal{X}^{j} \subset \mathcal{X}^{j+1}$ is both $\tilde{\Lambda}$-equivariant and $j$-connected, i.e., its homotopy groups vanish in degree $\leq j$. Let $\mathcal{X}^{\infty}=\bigcup_{j=1}^{\infty} \mathcal{X}^{j}$ with the direct limit topology. The induced action of $\tilde{\Lambda}$ on $\mathcal{X}^{\infty}$ is free. Let $X^{j}$ be the orbit space of $\mathcal{X}^{j} \times M$ under the diagonal action of $\tilde{\Lambda}$, and set

$$
X^{\infty}=\underset{j}{\lim } X^{j}
$$

Let $\mathcal{B}$ be the orbit space $M / \tilde{\Lambda}$ and $p_{j}: X^{j} \rightarrow \mathcal{B}$ the map induced from the canonical projection of $\mathcal{X}^{j} \times M$ onto $M$. The set $\left\{p_{j}\right\}_{j=1}^{\infty}$ is a collection of stratified systems of fiber bundles on $\mathcal{B}$ in the sense described above. For our ensuing applications we will have to define a homotopy class of maps $p_{\infty}: X^{\infty} \rightarrow \mathcal{B}$; in the following we write $X, p$ for $X^{\infty}, p_{\infty}$ in order to simplify notation.

Stratified string fields and branes. In Type II string theory on the hyperbolic orbifold background $\mathcal{B}$, one needs to endow $\mathcal{B}$ with extra geometric data, such as a Spin structure; the manifold $\mathcal{B}$ admits Spin structures if and only if its first and second Stiefel-Whitney classes vanish. In what follows we shall analyse to what extent this is compatible with stability and the stratification structure of $p: X \rightarrow \mathcal{B}$. In the worldsheet theory this is accomplished as follows.

Definition 2.1 Let $p: X \rightarrow \mathcal{B}$ be a collection of stratified fiber bundles over a hyperbolic orbifold. A Type II stratified string orbifold is a quadruple $(\Sigma, \phi ; \widehat{\Sigma}, \widehat{\phi})$ consisting of a string field $\phi: \Sigma \rightarrow \mathcal{B}$, a stratified system of fiber bundles $\widehat{p}: \widehat{\Sigma} \rightarrow \Sigma$ endowed with a Spin structure, and a stratum preserving lift of $\phi$ to an equivariant map $\widehat{\phi}: \widehat{\Sigma} \rightarrow X$ of the same homotopy 
type such that the diagram of maps of stacks $\widehat{\Sigma} \rightarrow \mathcal{B}$

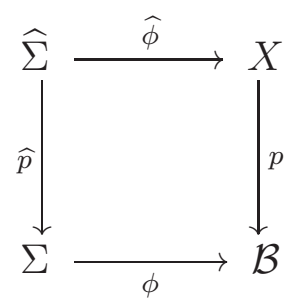

commutes.

Let us unpack Def.2.1. As before, the string field $\phi: \Sigma \rightarrow \mathcal{B}$ is an oriented principal $\tilde{\Lambda}$-bundle $P \rightarrow \Sigma$ and a $\tilde{\Lambda}$-equivariant map $P \rightarrow M$, and similarly $\widehat{\phi}: \widehat{\Sigma} \rightarrow X$ is a principal $\tilde{\Lambda}$-bundle $\widehat{P} \rightarrow \widehat{\Sigma}$ and a $\tilde{\Lambda}$-equivariant map $\widehat{P} \rightarrow \mathcal{X}^{\infty} \times M$; the pullback by $\widehat{p}: \widehat{\Sigma} \rightarrow \Sigma$ induces a morphism of principal $\tilde{\Lambda}$-bundles $\widehat{p}^{*}: P \rightarrow \widehat{P}$. If the stratification of the worldsheet is $\Sigma=\coprod_{i} \Sigma_{i}$, then commutativity of the diagram (2.2) implies the stratum preserving property $\phi\left(\Sigma_{i}\right)=(p \circ \widehat{\phi})\left(\widehat{p}^{-1}\left(\Sigma_{i}\right)\right) \subseteq \mathcal{B}_{i}$. The collection of all quadruples $(\Sigma, \phi ; \widehat{\Sigma}, \widehat{\phi})$ forms a groupoid in the obvious way; they provide the worldsheet "probes" of the structure of the background $\mathcal{B}$.

In the particular instance where $M$ involves hyperbolic space forms $H^{n}$, supersymmetry (or more generally stability) of the background $\mathcal{B}$ and the brane charges it supports can be analysed as discussed above. In Sect. 4 we shall develop stability conditions for branes in these backgrounds. Our new impetus will be a modification of the usual spectral sequence extension problem by regarding $\mathcal{B}$ as the base of a stratified fiber bundle, which incorporates the stratifications $\widehat{\phi}$ of open string fields from Def. 2.1 when the worldsheet $\Sigma$ has a boundary $\partial \Sigma \neq \varnothing$; the existence of the equivariant lift $\widehat{\phi}$ already constrains the homology of $\Sigma$. For this, we shall propose that branes in these backgrounds are classified by a suitable homology theory on the category of spaces with stratified systems of fiber bundles (in the sense of the Eilenberg-Steenrod axioms for homology). This is the spectral sheaf homology of Sect. 3 below which defines a functor of simplicial maps $p: X \rightarrow \mathcal{B}$; we think of $p$ as defining a "local coefficient system" over $\mathcal{B}$ and define the associated twisted homology, which has a concrete realization in terms of chain complexes. Amongst other features, we shall see that the resulting stability conditions play an important role in assessing the stability of the hyperbolic orbifold backgrounds discussed above.

\section{Quinn homology}

Homology loop spectra. We shall explain in some generality the homotopy theoretic approach to calculating the pertinent homology groups which arise in the following; see [19] for further definitions and discussion. In this paper we shall always assume that the background $\mathcal{B}$ is a simply connected finite-dimensional $\mathrm{CW}$-complex. For the present discussion we assume for simplicity that $\mathcal{B}$ is compact, otherwise it should be replaced by its one-point compactification $\mathcal{B}_{+}$in all considerations below. Let $\mathcal{B}_{0}$ be a subspace of $\mathcal{B}$. Let $\mathcal{G}=\left\{\mathcal{G}_{i}\right\}_{i \in \mathbb{Z}}$ 
be an arbitrary loop spectrum with canonical homotopy equivalences of based CW-spaces $\mathcal{G}_{i} \rightarrow \Omega \mathcal{G}_{i+1}$, where $\Omega$ denotes the based loop space.

Then the (generalized) homology groups for the finite $\mathrm{CW}$-pair $\left(\mathcal{B}, \mathcal{B}_{0}\right)$ with coefficients in $\mathcal{G}$ are defined for $k \in \mathbb{Z}$ by 20 ]

$$
H_{k}\left(\mathcal{B}, \mathcal{B}_{0} ; \mathcal{G}\right)=\underset{j}{\lim } \pi_{j+k}\left(\left(\mathcal{B} / \mathcal{B}_{0}\right) \wedge \mathcal{G}_{j}\right)
$$

where the factor space $X \wedge Y:=X \times Y / X \vee Y$ is the smash product of two spaces $X$ and $Y$ with base points $x_{0}$ and $y_{0}$ which is obtained from the product $X \times Y$ by collapsing the subspace $\left(X \times\left\{y_{0}\right\}\right) \cup\left(\left\{x_{0}\right\} \times Y\right)$ to a single point. For the directed limit (3.1) (which is a categorical colimit in the category of spectra) one uses the map

$$
\pi_{j+k}\left(\left(\mathcal{B} / \mathcal{B}_{0}\right) \wedge \mathcal{G}_{j}\right) \longrightarrow \pi_{j+k+1}\left(\left(\mathcal{B} / \mathcal{B}_{0}\right) \wedge \mathcal{G}_{j+1}\right)
$$

induced by the mapping $\Sigma \mathcal{G}_{j} \rightarrow \mathcal{G}_{j+1}$ as the composition

$$
\begin{aligned}
\pi_{j+k}\left(\left(\mathcal{B} / \mathcal{B}_{0}\right) \wedge \mathcal{G}_{j}\right) \stackrel{\Sigma}{\longrightarrow} & \pi_{j+k+1}\left(\Sigma\left(\left(\mathcal{B} / \mathcal{B}_{0}\right) \wedge \mathcal{G}_{j+1}\right)\right) \\
\| & \pi_{j+k+1}\left(\left(\mathcal{B} / \mathcal{B}_{0}\right) \wedge \Sigma \mathcal{G}_{j}\right) \longrightarrow \pi_{j+k+1}\left(\left(\mathcal{B} / \mathcal{B}_{0}\right) \wedge \mathcal{G}_{j+1}\right)
\end{aligned}
$$

where for a space $X$ the suspension $\Sigma X$ is the space obtained from the product $X \times \Delta^{1}$ by collapsing the subspaces $X \times\{0\}$ and $X \times\{1\}$ to single points, and we have used the continuous structure maps $\mathcal{G}_{j} \rightarrow \Omega \mathcal{G}_{j+1}$.

One can alternatively define the homology groups $H_{\sharp}\left(\mathcal{B}, \mathcal{B}_{0} ; \mathcal{G}\right)$ directly as the homotopy groups of a loop spectrum $\left(\mathcal{B} / \mathcal{B}_{0}\right) \otimes \mathcal{G}$ by setting

$$
\left(\left(\mathcal{B} / \mathcal{B}_{0}\right) \otimes \mathcal{G}\right)_{i}=\underset{j}{\lim _{j}} \Omega^{j}\left(\left(\mathcal{B} / \mathcal{B}_{0}\right) \wedge \mathcal{G}_{i+j}\right)
$$

where the maps in the directed system are induced from the structure maps $\mathcal{G}_{i} \rightarrow \Omega \mathcal{G}_{i+1}$ of the loop spectrum $\mathcal{G}$.

In general the relation between homotopy and homology functors is very subtle, even though there are natural homomorphisms $\pi_{j}(\mathcal{B}) \rightarrow H_{j}(\mathcal{B})$; for example, for $j \geq 1$ there is the Hurewicz homomorphism $\pi_{j}(\mathcal{B}) \rightarrow H_{j}(\mathcal{B} ; \mathbb{Z})$. While the homology groups $H_{j}(\mathcal{B})$ are relatively computable, the homotopy groups $\pi_{j}(\mathcal{B})$ are typically not as there is no analogue of the Mayer-Vietoris sequence that is implied by the Eilenberg-Steenrod axioms for homology theories. This means that the homology groups defined in this way are in general difficult to compute explicitly.

Spectral sheaves. Following [21] let us consider a fiber bundle over $\mathcal{B}$ with section

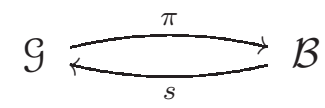


so that $\pi \circ s=\operatorname{id}_{\mathcal{B}}$. The loop space $\Omega_{\mathcal{B}}(\mathcal{G})$ over $\mathcal{B}$ is the space of maps $F: \mathcal{B} \times \Delta^{1} \rightarrow \mathcal{G}$ which commute with the projection $\pi$ to $\mathcal{B}$, and which coincide with the section $s$ when restricted to $\mathcal{B} \times\{0\}$ and $\mathcal{B} \times\{1\}$. Then $\Omega_{\mathcal{B}}(\mathcal{G})$ is again a fiber bundle over $\mathcal{B}$ with section and the fibers of $\Omega_{\mathcal{B}}(\mathcal{G}) \rightarrow \mathcal{B}$ are loop spaces of the fibers of $\mathcal{G} \rightarrow \mathcal{B}$. A spectral sheaf is a sequence of fiber bundles with section $\left\{\mathcal{G}_{j}\right\}_{j \in \mathbb{Z}}$ and morphisms $\alpha_{j}: \mathcal{G}_{j} \rightarrow \Omega_{\mathcal{B}}\left(\mathcal{G}_{j+1}\right)$. For a subspace $\mathcal{B}_{0} \subset \mathcal{B}$, these structure maps define maps [5]

$$
\mathcal{G}_{j} / \iota(\mathcal{B}) \cup \pi^{-1}\left(\mathcal{B}_{0}\right) \stackrel{\alpha_{j}}{\longrightarrow} \Omega_{\mathcal{B}}\left(\mathcal{G}_{j+1}\right) / s(\mathcal{B}) \cup \pi^{-1}\left(\mathcal{B}_{0}\right) \longrightarrow \Omega\left(\mathcal{G}_{j+1} / s(\mathcal{B}) \cup \pi^{-1}\left(\mathcal{B}_{0}\right)\right),
$$

where the second map is the inclusion of based loop spaces and $s(\mathcal{B}) \cup \pi^{-1}\left(\mathcal{B}_{0}\right)$ is taken to the base point. Then there is a homology loop spectrum defined by

$$
\mathcal{Q}_{k}\left(\mathcal{B}, \mathcal{B}_{0} ; \mathcal{G}\right)=\underset{j}{\lim } \Omega^{j}\left(\mathcal{G}_{k+j} / s(\mathcal{B}) \cup \pi^{-1}\left(\mathcal{B}_{0}\right)\right),
$$

and the Quinn homology groups $Q_{\sharp}\left(\mathcal{B}, \mathcal{B}_{0} ; \mathcal{G}\right)$ are the homotopy groups of this spectrum [5]; the structure maps on $\mathcal{Q}_{k}\left(\mathcal{B}, \mathcal{B}_{0} ; \mathcal{G}\right)$ are induced by those for $\mathcal{G}$.

Spectral sequences. Let us now consider the Atiyah-Hirzebruch type spectral sequence with constant coefficients which is derived in [22]. For this, we note that any spectrum $\mathcal{G}$ has a natural $j$-connected cover $\mathcal{G}^{(j)} \rightarrow \mathcal{G}$. Applying this construction to the fibers of a spectral sheaf $\mathcal{G} \rightarrow \mathcal{B}$ gives a sequence of spectral sheaves

$$
\cdots \longleftarrow \mathcal{G}^{(j)} \longleftarrow \mathcal{G}^{(j+1)} \longleftarrow \cdots
$$

which we can regard as a filtration of $\mathcal{G}$. Applying the homotopy functor $\pi_{\sharp}$ to the filtration $(\underline{3.3})$ gives a spectral sequence which converges to the spectral sheaf homology $Q_{\sharp}(\mathcal{B} ; \mathcal{G})$. The $E^{2}$ terms are given by

$$
E_{i, j}^{2}=H_{i}\left(\mathcal{B} ; \pi_{j} \mathcal{G}\right)
$$

because the fiber over $\mathcal{B}$ of $\mathcal{G}^{(j+1)} \rightarrow \mathcal{G}^{(j)}$ is the spectral sheaf $\Omega_{\mathcal{B}}^{-j-2}\left(\underline{K}\left(\pi_{j+1} \mathcal{G}, \sharp\right)\right)$; here $\underline{K}(-, \sharp)$ is the fiberwise Eilenberg-MacLane spectrum functor from abelian groups to loop spectra which corepresents the homology functor, i.e., for any abelian group $\Lambda$ one has $H_{i}(\mathcal{B} ; \Lambda)=\pi_{i}(\mathcal{B} \wedge \mathcal{H})$ for the spectrum $\mathcal{H}=\left\{\mathcal{H}_{i}\right\}_{i \in \mathbb{Z}}$ defined by taking $\mathcal{H}_{i}=\underline{K}(\Lambda, i)$. This functor will play an important role again in Sect. 5 .

In our specific applications to follow all spectra considered are those of C̆ech-type theories, and thus there are isomorphisms between homomorphisms in these theories. The homologies of these spectra with coefficients in sheaves are isomorphic in a unique way, and in particular all constructions for Čech homology are in one-to-one correspondence with those of singular homology. Hence the differentials of the spectral sequences can be gleamed from those of singular homology.

\section{Branes in spaces stratified fibered over orbifolds}

Homology with stratified coefficients. Suppose that the background $\mathcal{B}$ is filtered by closed subsets $\mathcal{B}^{i} \subset \mathcal{B}^{i+1}, i \geq 1$. A stratified system of groups $A$ over $\mathcal{B}$ consists of 
neighborhoods $U_{i}$ of the strata $\mathcal{B}_{i}=\mathcal{B}^{i}-\mathcal{B}^{i-1}$, locally constant systems of groups $A_{i}:=A\left(U_{i}\right)$ over $U_{i}$, and for each $i>j$ a homomorphism $\theta_{i j}: A_{i} \rightarrow A_{j}$ over $U_{i} \cap U_{j}$ such that if $i>j>k$ then $\theta_{j k} \circ \theta_{i j}=\theta_{i k}$ over $U_{i} \cap U_{j} \cap U_{k}$.

Let $\mathcal{G}$ be a spectrum-valued homotopy invariant functor on the category of spaces, and let $\mathcal{G}(Y)$ be the stable topological pseudo-isotopy loop spectrum associated to a topological space $Y$; a covariant functor $\mathcal{G}$ from spaces to spectra is homotopy invariant if a homotopy equivalence of spaces $X \cong Y$ induces a homotopy equivalence of spectra $\mathcal{G}(X) \cong \mathcal{G}(Y)$ [5]. Then to a stratified system of fiber bundles $p: X \rightarrow \mathcal{B}$ one can associate the Quinn homology loop spectrum $\mathcal{Q}(\mathcal{B} ; \mathcal{G}(p))$ with twisted spectrum coefficients and a map of loop spectra $\mathcal{Q}(\mathcal{B} ; \mathcal{G}(p)) \rightarrow \mathcal{G}(X)$, where $\mathcal{G}(p)$ is the spectral sheaf over $\mathcal{B}$ obtained by applying $\mathcal{G}$ to the fibers of $p$ [5]. One can construct an Atiyah-Hirzebruch type spectral sequence in the following way [5, 23]. The homotopy groups $\pi_{j} \mathcal{G}\left(p^{-1}(b)\right)$ corresponding to the point $b \in \mathcal{B}$ form stratified systems of abelian groups over $\mathcal{B}$, which we denote by $\pi_{j} \mathcal{G}(p)$. In this case the filtration of $\mathcal{B}$ gives a (homological) spectral sequence with

$$
E_{i, j}^{2}=H_{i}\left(\mathcal{B} ; \pi_{j} \mathcal{G}(p)\right)
$$

which converges to the spectral sheaf homology groups $Q_{i+j}(\mathcal{B} ; \mathcal{G}(p))=\pi_{i+j} \mathcal{Q}(\mathcal{B} ; \mathcal{G}(p))$. In the case of string orbifolds, i.e., when the stratified system of fiber bundles is given by a group action as in Sect. 2, this spectral sequence agrees with that of [24]. In particular, in this instance the spectral sheaf homology is isomorphic to Bredon equivariant homology [18] which naturally realises stringy orbifold cohomology and stability conditions for fractional D-branes on orbifolds [25].

Let $A$ be a stratified system of abelian groups over $\mathcal{B}$. The homology groups $H_{j}(\mathcal{B} ; A)$ can be calculated as follows. A compact background $\mathcal{B}$ can be triangulated by a simplicial complex, which is a finite family of closed subsets $\left\{\mathcal{T}_{j}\right\}_{j=0}^{n}$ which cover $\mathcal{B}$ together with a family of homeomorphisms $\varphi_{j}: \Delta^{j} \rightarrow \mathcal{T}_{j}$ where each $\Delta^{j} \subset \mathbb{R}^{n}$ is a Euclidean $j$-simplex; we assume that each stratum $\mathcal{T}_{j}$ is a subcomplex. Then a stratified system $A$ restricted to each open simplex is a constant system of coefficients and

$$
H(\mathcal{B} ; A)=\bigoplus_{j \geq 0} H_{j}(\mathcal{B} ; A)
$$

is the homology of the finite chain complex

$$
C_{0}(\mathcal{B} ; A) \stackrel{\partial_{1}}{\longleftarrow} C_{1}(\mathcal{B} ; A) \stackrel{\partial_{2}}{\longleftarrow} \cdots \stackrel{\partial_{n}}{\longleftarrow} C_{n}(\mathcal{B} ; A), \quad C_{j}(\mathcal{B} ; A)=\bigoplus_{\sigma \in \mathcal{T}_{j}} A(\sigma)
$$

where the simplicial boundary homomorphisms $\partial_{j}$ are defined by decomposing a $j$-simplex $\sigma \in \mathcal{T}_{j}$ into $(j+1)$ ! simplices of smaller dimension; the vertices of the new simplices are the centers of gravity of the faces of the original simplex.

$\boldsymbol{E}_{\mathbf{0}, \mathbf{j}}^{\mathbf{2}}$-terms. The $E_{0, j}^{2}$-terms in the spectral sequence (4.1) can be evaluated in the following manner. Let $\Pi$ be the extension of the fundamental group $\pi_{1}(M)$ determined by the action of $\tilde{\Lambda}$ on $M$; the group $\Pi$ is isomorphic to the factor group $\pi_{1}(X) / \pi_{1}\left(\mathcal{X}^{\infty}\right)$. Let $\mathcal{F}(\Pi)$ be the 
category with objects the finite subgroups of $\Pi$; for each element $\beta \in \Pi$ we can determine a morphism $B_{1} \rightarrow B_{2}$ of objects of $\mathcal{F}(\Pi)$ if $\beta B_{1} \beta^{-1} \subseteq B_{2}$. Then the $E_{0, j}^{2}$-terms are isomorphic to the direct limit

$$
E_{0, j}^{2} \cong \underset{B \in \mathcal{F}(\Pi)}{\lim _{j}} \pi_{j} \mathcal{G}\left(\mathcal{X}^{\infty} / \mathfrak{p}(B)\right)
$$

where $\mathfrak{p}: \Pi \rightarrow \tilde{\Lambda}$ is the canonical projection and $\mathcal{X}^{\infty} / \mathfrak{p}(B)$ are the corresponding orbit spaces. This isomorphism can be proven by using a basic result of E. Cartan [26].

Strongly virtually negatively curved groups. Let us choose a spectrum-valued functor of spaces $\mathcal{G}$ with homotopy groups $\pi_{i} \mathcal{G}(X)=\widetilde{K}_{i}(\mathbb{Z} \Lambda)$, where $\Lambda:=\pi_{1}(X)$. In the case when $\mathcal{X}^{\infty}$ is contractible one has isomorphisms [23]

$$
K_{i}(\mathbb{Z} \Lambda) \otimes \mathbb{Q} \cong \bigoplus_{j \geq 0} H_{j}\left(\mathcal{B} ; K_{i-j}\left(\mathbb{Z} \Lambda_{b}\right) \otimes \mathbb{Q}\right)
$$

where $\Lambda_{b}=\pi_{1}\left(p^{-1}(b)\right)$ for $b \in \mathcal{B}$. Similarly, by choosing $\pi_{i} \mathcal{G}(X)=W h_{i}(\Lambda)$ one has [23]

$$
W h_{i}(\Lambda) \otimes \mathbb{Q} \cong \bigoplus_{j \geq 0} H_{j}\left(\mathcal{B} ; W h_{i-j}\left(\Lambda_{b}\right) \otimes \mathbb{Q}\right)
$$

where the Whitehead groups $W h_{i}(\Lambda)$ are the homotopy groups of the cofiber of the map $\mathcal{Q}(\underline{B} \Lambda ; \mathcal{K}(\mathbb{Z} \Lambda)) \rightarrow \mathcal{K}(\mathbb{Z} \Lambda)$ between spectral sheaf homology and algebraic K-theory spectra. Here $W h_{i-j}\left(\Lambda_{b}\right) \otimes \mathbb{Q}$ and $K_{i-j}\left(\mathbb{Z} \Lambda_{b}\right) \otimes \mathbb{Q}$ form stratified systems of abelian groups over $\mathcal{B}$. We therefore say that a group $\Lambda$ is strongly virtually negatively curved if it is isomorphic to the fundamental group $\pi_{1}(X)$ when $\mathcal{X}^{\infty}$ is contractible.

For example, any discrete cocompact subgroup $\Lambda$ of $G$, where $G$ is one of the connected noncompact simple split rank one Lie groups with finite center $O(n, 1), U(n, 1), S p(n, 1)$, or $F_{4}$, is strongly virtually negatively curved $[23$. Let $\Gamma \subset G$ be a maximal compact subgroup; then $G / \Gamma$ is an irreducible non-compact symmetric space of rank one which up to local isomorphism can be represented as one of the quotients $H^{n}=S O_{1}(n, 1) / S O(n), S U(n, 1) / U(n)$, $\left((S p(n, 1) / S p(n)) \times S p(1), F_{4(20)} / \operatorname{Spin}(9)\right.$ of dimensions $n, 2 n, 4 n, 16$ respectively. The double coset space $\mathcal{B}:=\Lambda \backslash G / \Gamma$ is a locally symmetric space which is in particular a compact Riemannian manifold with fundamental group $\Lambda$. The algebraic K-theory of $\Lambda$ can thus be calculated in terms of the stratified homology of $\mathcal{B}$ through the isomorphisms [23]

$$
K_{i}(\mathbb{Z} \Lambda) \otimes \mathbb{Q} \cong \bigoplus_{j \geq 0} H_{j}\left(\mathcal{B} ; \mathcal{V}_{i-j}\right)
$$

where $\mathcal{V}_{i}$ is a stratified system of $\mathbb{Q}$-vector spaces over $\mathcal{B}$ such that the vector space $\mathcal{V}_{i}(\Lambda g \Gamma)$ corresponding to the double coset $\Lambda g \Gamma$ for $g \in G$ is isomorphic to $K_{i}\left(\mathbb{Z}\left(\Lambda \cap g \Gamma g^{-1}\right)\right) \otimes \mathbb{Q}$; as previously $\Lambda \cap\left(g \Gamma g^{-1}\right)$ is a finite subgroup of $\Lambda$ because $\Gamma$ is compact.

Stability conditions for D-branes. Let us now analyse D-brane stability in this setting. We introduce D-branes into these backgrounds following the definitions and conventions of [27]. K-homology cycles encode data that must be carried by any D-brane, such as a 
$\operatorname{Spin}^{\mathbb{C}}$ structure and a complex vector bundle. A D-brane on a background $\mathcal{B}$ of Type II string theory is specified by a triple $(\mathcal{W}, F, \psi)$, where $\mathcal{W}$ is a $\operatorname{Spin}^{\mathbb{C}}$ manifold regarded as a brane worldvolume, $F$ is the Chan-Paton bundle on $\mathcal{W}$ with $[F] \in K^{0}(\mathcal{W})$ and $\psi: \mathcal{W} \rightarrow \mathcal{B}$ is a continuous map. D-brane charges can then be regarded as homology classes of K-cycles $[\mathcal{W}, F, \psi] \in K_{\sharp}(\mathcal{B})$. The equivalence relation of vector bundle modification in K-homology identifies pairs of D-branes for which one is a spherical fiber bundle over the other, in accordance with our treatment of the target space $\mathcal{B}$ as the base of a system of fiber bundles.

Any free brane (with no lower or higher brane charges) can wrap a homologically nontrivial cycle in $\mathcal{B}$; a D-brane $[\mathcal{W}, F, \psi]$ wraps $\mathcal{M} \subseteq \mathcal{B}$ if $\psi(\mathcal{W}) \subseteq \mathcal{M}$. The Atiyah-Hirzebruch spectral sequence provides a mathematical algorithm that determines which homology classes lift non-trivially to K-homology classes, i.e., it determines which D-branes are unstable and not allowed; in this instance one chooses a spectrum-valued functor of spaces $\mathcal{G}$ such that $\mathcal{G}(X)$ is homotopy equivalent to the Bott spectrum $\mathcal{K}=\left\{\mathcal{K}_{i}\right\}_{i \in \mathbb{Z}}$ defined by taking $\mathcal{K}_{i}$ to be the infinite unitary group $U(\infty)$ for $i$ odd and $\mathbb{Z} \times \underline{B} U(\infty)$ for $i$ even. For other flavors of branes one could choose the loop spectrum $\mathcal{G}(X)$ appropriate to the generalized homology theory which classifies those branes, although we shall see that D-branes (in the sense defined above) fit most naturally into the present framework. The spectral sequence keeps track of the possible obstructions for a homology cycle to survive to $E_{i, j}^{\infty}$, starting from $H_{i}\left(\mathcal{B} ; \pi_{j} \mathcal{G}(p)\right)$ in Eq. (4.1); the initial terms are given in Eq. (4.2). Given a brane wrapping a cycle in $\mathcal{B}$ and carrying a stratified system of groups (or local coefficient systems), we can ask if it is stable in the sense that it has a lift to a non-trivial class in the spectral sheaf homology $Q_{\sharp}(\mathcal{B} ; \mathcal{G}(p))$. We analyse this obstruction problem in the case that $p: X \rightarrow \mathcal{B}$ is simplicially stratified.

The first term of the spectral sequence is the relative Quinn homology

$$
E_{i, j}^{1}=Q_{i+j}\left(\mathcal{B}^{i}, \mathcal{B}^{i-1} ; \mathcal{G}(p)\right)
$$

where $\mathcal{G}(p)$ is the disjoint union of $\mathcal{G}\left(p^{-1}(\sigma)\right) \times \sigma$ over all simplices $\sigma$ of $\mathcal{B}$ modulo the equivalence relation which identifies $\mathcal{G}\left(p^{-1}\left(\partial_{j} \sigma\right)\right) \times \partial_{j} \sigma$ with its image in $\mathcal{G}\left(p^{-1}(\sigma)\right) \times \sigma$. Note that the natural projections $\mathcal{G}\left(p^{-1}(\sigma)\right) \times \sigma \rightarrow \sigma$ fit together to give a projection $\pi: \mathcal{G}(p) \rightarrow \mathcal{B}$, while the basepoints of the pieces fit together to form sections $s_{j}: \mathcal{B} \rightarrow \mathcal{G}_{j}(p)$ for each $j \in \mathbb{Z}$; the structure maps of the spectra $\mathcal{G}\left(p^{-1}(\sigma)\right)$ also fit together to give structure maps on $\mathcal{G}(p)$ that commute with the sections and projections, and hence $\mathcal{G}(p)$ is a spectral sheaf over $\mathcal{B}$. The first differential

$$
\mathrm{d}_{i, j}^{1}: E_{i, j}^{1} \longrightarrow E_{i-1, j}^{1}
$$

is the composition of the simplicial boundary homomorphism $\partial_{i}: Q_{i+j}\left(\mathcal{B}^{i}, \mathcal{B}^{i-1} ; \mathcal{G}(p)\right) \rightarrow$ $Q_{i+j-1}\left(\mathcal{B}^{i-1} ; \mathcal{G}(p)\right)$ with the pushforward $\kappa_{*}: Q_{i+j-1}\left(\mathcal{B}^{i-1} ; \mathcal{G}(p)\right) \rightarrow Q_{i+j-1}\left(\mathcal{B}^{i-1}, \mathcal{B}^{i-2} ; \mathcal{G}(p)\right)$ induced by the inclusion $\kappa:\left(\mathcal{B}^{i-1}, \varnothing\right) \hookrightarrow\left(\mathcal{B}^{i-1}, \mathcal{B}^{i-2}\right)$. The group $E_{i, j}^{1}$ parameterizes spectral sheaf homology classes on the $i$-skeleton of $\mathcal{B}$ which are trivial on the $i$ - 1 -skeleton; it classifies branes wrapping $i$-cycles of the stratified system of fiber bundles $p: X \rightarrow \mathcal{B}$ which carry no lower or higher degree brane charges. Alternatively, using excision we may write the group (4.4) as the reduced homology

$$
E_{i, j}^{1}=\widetilde{Q}_{i+j}\left(\mathcal{B}_{i} ; \mathcal{G}(p)\right):=Q_{i+j}\left(\mathcal{B}_{i}, \mathrm{pt} ; \mathcal{G}(p)\right)
$$


which classifies branes on the $i$-th stratum $\mathcal{B}_{i}$ of $p: X \rightarrow \mathcal{B}$ which carry no lower charges; such branes further support ordinary fiber bundles $p \mid: p^{-1}\left(\mathcal{B}_{i}\right) \rightarrow \mathcal{B}_{i}$ in addition to their usual Chan-Paton vector bundle which generalizes the roles of image branes of "fractional branes" on the covering space of an orbifold. For the simplicial stratification, $\mathcal{B}_{i}$ is a disjoint union of boundaries $\partial_{i} \sigma$ of simplicial cells $\sigma \in \mathcal{T}_{i}$, each of whose reduced homology can be computed using excision as the reduced homology of a sphere $\widetilde{Q}_{i+j}\left(S^{i} ; \mathcal{G}(p)\right) \cong Q_{j}(\mathrm{pt} ; \mathcal{G}(p))=$ $\pi_{j} \mathcal{G}(p)$ [24]. It then follows from the Eilenberg-Steenrod additivity axiom that the homology group (4.4) can be identified with the group of singular $i$-chains

$$
E_{i, j}^{1}=C_{i}\left(\mathcal{B} ; \pi_{j} \mathcal{G}(p)\right) \text {. }
$$

The second term of the spectral sequence is the homology of the differential $\mathrm{d}^{1}$ and is given by Eq. (4.1); by our previous results we can compute this term from the spectral sheaf homology groups $Q_{i}\left(\mathcal{B} ; \mathcal{K}\left(\mathbb{Z} \Lambda_{b}\right)\right)$ corresponding to algebraic K-theory, where up to conjugation each $\Lambda_{b}$ forms a sheaf of groups over $\mathcal{B}$. On the $r$-th term $E_{i, j}^{r}$, the differential $\mathrm{d}_{i, j}^{r}$ has bidegree $(-r, r-1)$ and $E_{i, j}^{r+1}$ is the corresponding homology group. The $E^{\infty}$ term is the inductive limit

$$
E_{i, j}^{\infty}=\underset{r}{\lim } E_{i, j}^{r}
$$

If $n=\operatorname{dim}(\mathcal{B})$, then $E_{i, j}^{r}=E_{i, j}^{\infty}$ for all $r>n$ and convergence of the spectral sequence means that there exists an ascending filtration $F_{m, m-i} Q_{m}(\mathcal{B} ; \mathcal{G}(p))$ of $Q_{m}(\mathcal{B} ; \mathcal{G}(p))$, with $0 \leq m \leq n$, such that

$$
F_{i, j} Q_{i+j}(\mathcal{B} ; \mathcal{G}(p)) / F_{i-1, j+1} Q_{i+j}(\mathcal{B} ; \mathcal{G}(p)) \cong E_{i, j}^{\infty} .
$$

If $\imath: \mathcal{B}^{i} \hookrightarrow \mathcal{B}$ denotes the inclusion of the $i$-skeleton in $\mathcal{B}$, then the filtration groups

$$
F_{i, j} Q_{i+j}(\mathcal{B} ; \mathcal{G}(p))=\operatorname{im}\left(\imath_{*}: Q_{i+j}\left(\mathcal{B}^{i} ; \mathcal{G}(p)\right) \rightarrow Q_{i+j}(\mathcal{B} ; \mathcal{G}(p))\right)
$$

consist of branes in $\mathcal{B}$ wrapping cycles supported in the $i$-skeleton, whereas the extension groups (4.5) consist of branes in the $i$-skeleton which are not supported on the $i$-1-skeleton, i.e., $E_{i, j}^{\infty}$ consists of $i-1$-branes which carry no lower brane charges.

Let $\mathcal{M} \subseteq \mathcal{B}$ be a compact $i$-dimensional submanifold without boundary in $p: X \rightarrow \mathcal{B}$ which defines a non-trivial cycle $[\mathcal{M}] \in E_{i, j}^{2}$ in Eq. (4.1). In the case that $[\mathcal{M}]$ extends through the spectral sequence as a non-trivial element of $E_{i, j}^{\infty}$ and hence has a non-trivial lift to Khomology, there exists a stable D-brane $[\mathcal{W}, F, \psi]$ wrapping $\mathcal{M}$ on the $i$-skeleton of $\mathcal{B}$ which carries no lower brane charges, i.e., $\psi(\mathcal{W}) \subseteq \mathcal{B}^{i}$. As the homotopy groups $\pi_{j} \mathcal{G}\left(p^{-1}(b)\right)$ form stratified systems of groups $\pi_{j} \mathcal{G}(p)$ over the background $\mathcal{B}$, D-branes wrapping spaces stratified fibered over hyperbolic orbifolds carry charges which induce new additive structures on K-homology due to the stratification from $E_{i, j}^{\infty}$ in the solution of the extension problem required to get the filtration groups (4.6). Cycles with $[\mathcal{M}] \notin \operatorname{ker}\left(\mathrm{d}_{i, j}^{r}\right)$ correspond to anomolous D-branes, while if $[\mathcal{M}] \in \operatorname{im}\left(\mathrm{d}_{i, j}^{r}\right)$ for some $r$ then the homology class $[\mathcal{M}]$ can be lifted to K-homology but this lift is trivial as it vanishes in $E_{i, j}^{\infty}$ : In this case there exists a D-brane wrapping $\mathcal{M}$ in the $i$-th stratum $\mathcal{B}_{i}$ with no lower brane charges, but this D-brane is unstable. 
Examples. (i) Recalling that the differentials can be gleamed from singular homology (cf. Sect. 3), it follows that $\mathrm{d}_{i, j}^{r}=0$ for all $r$ even [28] and the first non-trivial condition for a homology class $[\mathcal{M}] \in E_{i, 0}^{2}$ to survive to $E_{i, 0}^{\infty}$ is given by

$$
\mathrm{d}_{i, 0}^{3}[\mathcal{M}]=0 .
$$

This includes the Poincaré dual of the condition that the normal bundle $N \mathcal{M}$ to $\mathcal{M}$ in $\mathcal{B}$ is a $\operatorname{Spin}^{\mathbb{C}}$ vector bundle [28], i.e., that $W_{3}(N \mathcal{M})=0$, where $W_{3}(N \mathcal{M}) \in H^{3}(\mathcal{M} ; \mathbb{Z})$ is the canonical integral lift of the third Stiefel-Whitney class $w_{3}(N \mathcal{M}) \in H^{3}\left(\mathcal{M} ; \mathbb{Z}_{2}\right)$ of $N \mathcal{M}$ which is the torsion class defined as $W_{3}(N \mathcal{M})=\beta\left(w_{2}(N \mathcal{M})\right)$, where $\beta: H^{2}\left(\mathcal{M} ; \mathbb{Z}_{2}\right) \rightarrow$ $H^{3}(\mathcal{M} ; \mathbb{Z})$ is the Bockstein homomorphism. Applying the condition (4.7) to a D-brane $[\mathcal{W}, F, \psi]$ wrapping $\mathcal{M}$ requires the normal bundle for the tangent bundle $T \mathcal{W}$ with respect to the map $\psi: \mathcal{W} \rightarrow \mathcal{B}$ to be $\operatorname{Spin}^{\mathbb{C}}$; this is the real vector bundle $N \mathcal{W} \rightarrow \mathcal{W}$ such that $T \mathcal{W} \oplus N \mathcal{W}$ is isomorphic to the pullback by $\psi$ of a $\operatorname{Spin}^{\mathbb{C}}$ vector bundle over $\mathcal{B}$ (recall that $\mathcal{W}$ is already a $\operatorname{Spin}^{\mathbb{C}}$ manifold, i.e., a $\operatorname{Spin}^{\mathbb{C}}$ lift of $T \mathcal{W}$ exists). Such a choice of Korientation plays a crucial role in determining the amount of supersymmetry which persists in the hyperbolic orbifold backgrounds of Sect. 2,

(ii) We can give a novel class of examples of unstable backgrounds which illustrates the destabilizing effects of the additional local fiber bundles over the strata of the hyperbolic orbifold $\mathcal{B}$. Let $\mathcal{G}(\mathcal{B} ; p)$ be the homotopy cofiber of the map $\mathcal{Q}(\mathcal{B} ; \mathcal{G}(p)) \rightarrow \mathcal{G}(X)$ in the category of spectra, which is also a loop spectrum. For each of the stratified systems of fiber bundles $p: X \rightarrow \mathcal{B}$ there is a homotopy equivalence of loop spectra $\mathcal{G}(X) \cong \mathcal{Q}(\mathcal{B} ; \mathcal{G}(p)) \times \mathcal{G}(\mathcal{B} ; p)$, which is compatible with the Atiyah-Hirzebruch type spectral sequence (4.1). Suppose that a stratified system of fiber bundles $p: X \rightarrow \mathcal{B}$ extends through the spectral sequence as a nontrivial element of the homology groups $E_{i, j}^{2}$. Then one can calculate the rational K-groups via [23, Thm. 2], which asserts that if $\mathcal{X}^{\infty}$ is aspherical with $W h_{i}\left(\pi_{1}\left(\mathcal{X}^{\infty} \times S^{1}\right)\right) \otimes \mathbb{Q}=0$ for all $i \in \mathbb{Z}$, then $\mathcal{G}(\mathcal{B} ; p) \otimes \mathbb{Q}=0$. Hence the stratified system of fiber bundles extends through the spectral sequence as a non-trivial element of the homology groups, but if the homology is torsion-free it vanishes in $E_{i, j}^{\infty}$ and thus has a trivial lift to K-homology. In this case there exists a D-brane wrapping $\mathcal{B}$, but this D-brane is unstable.

(iii) For strongly virtually negatively curved groups $\Lambda$, the groups $\Lambda_{b}=\pi_{1}\left(p^{-1}(b)\right)$ for $b \in \mathcal{B}$ are finite because they are isomorphic to subgroups of $\tilde{\Lambda}$. As a consequence one can use the wealth of results available for the algebraic K-theory of finite groups [29, 30] to formulate stability conditions for D-branes wrapping homology cycles. For example, if $\Lambda$ is strongly virtually negatively curved then $K_{n}(\mathbb{Z} \Lambda)=0$ for all $n<-1$, whereas

$$
K_{-1}(\mathbb{Z} \Lambda) \cong \underset{L \in \mathcal{F}(\Lambda)}{\lim _{L}} K_{-1}(\mathbb{Z} L)
$$

is an abelian group finitely generated by the images of $K_{-1}(\mathbb{Z} L)$, as $L$ varies over the finite subgroups of $\Lambda$, under the map functorially induced by the inclusion of $L$ into $\Lambda$.

(iv) Let $\Lambda \subset G$ be a discrete cocompact torsion-free subgroup as above, and $\Gamma \subset G$ a maximal compact subgroup; let $X=G / \Gamma$. In this case we can use the orbifold stratified fiber bundle $p: X \rightarrow \mathcal{B}$ to make the corresponding K-homology classes of D-branes on $\mathcal{B}=[\Lambda \backslash X]$ explicit. Given a finite-dimensional unitary representation $\rho$ of the fundamental 
group $\Lambda$ there corresponds a locally homogeneous Chan-Paton vector bundle $\mathcal{E}_{\rho}=\Lambda \backslash\left(X \times V_{\rho}\right)$ over $\mathcal{B}$, where the fiber $V_{\rho}$ of $\mathcal{E}_{\rho}$ is the representation space of $\rho$ and the $\Lambda$-action on $X \times V_{\rho}$ is defined by $\lambda \cdot(x, v):=(\lambda \cdot x, \rho(\lambda) v)$ for $(\lambda, x, v) \in \Lambda \times X \times V_{\rho}$. In the case that $X$ admits a $G$-invariant $\operatorname{Spin}^{\mathbb{C}}$ structure, let $\mathfrak{D}_{\rho}$ be the Dirac operator of $\mathcal{B}$ acting on smooth sections of the homogeneous spinor bundle $\mathcal{S}$ over $\mathcal{B}$ twisted by the vector bundle $\mathcal{E}_{\rho}$; it is obtained by projecting the Dirac operator $\mathfrak{D}$ of $X$ (which is $G$-invariant and hence $\Lambda$-invariant) to $\mathcal{B}$. As we discuss in Sect. 5, this defines an analytic K-homology class of $\mathcal{B}$. The properties of this Dirac operator can be studied by using the spherical harmonic analysis on the rank one symmetric space $X$ developed by [31, 32, 33] using Harish-Chandra's Plancherel density.

\section{Inclusion of $\boldsymbol{H}$-flux}

$\boldsymbol{B}$-fields and twisted K-theory. In the presence of a Neveu-Schwarz $B$-field whose curvature $H$ represents a non-trivial element $[H] \in H^{3}(\mathcal{B} ; \mathbb{Z})$, the Chan-Paton vector bundles on D-branes should be replaced by suitable "twisted" gauge bundles. Recall [34 that a twisted bundle $P_{H} \rightarrow \mathcal{B}$ is given by a collection of locally defined bundles of Hilbert spaces $E_{i} \rightarrow U_{i}$ such that $\left.P_{H}\right|_{U_{i}} \cong \mathbb{P}\left(E_{i}\right)$ with respect to an open cover $\left\{U_{i}\right\}$ of the background $\mathcal{B}$. The gluing functions between charts are realised by isomorphisms

$$
g_{i j}:\left.\left.E_{i}\right|_{U_{i} \cap U_{j}} \stackrel{\sim}{\longrightarrow} E_{j}\right|_{U_{i} \cap U_{j}} .
$$

On triple overlaps $U_{i} \cap U_{j} \cap U_{k}$ the composition $g_{i j} \circ g_{j k} \circ g_{k i}$ is multiplication by a function $f_{i j k}: U_{i} \cap U_{j} \cap U_{k} \rightarrow U(1)$; this data is equivalent to a lift $g_{i j}: U_{i} \cap U_{j} \rightarrow U(\mathcal{H})$ of the $P U(\mathcal{H})$-valued one-cocycle $\tilde{g}_{i j}: U_{i} \cap U_{j} \rightarrow P U(\mathcal{H})$ of the bundle $P_{H} \rightarrow \mathcal{B}$, where $P U(\mathcal{H})=U(\mathcal{H}) / U(1)$ is the group of projective unitary operators with the topology induced by the norm topology on the unitary group $U(\mathcal{H})$ of an infinite-dimensional separable Hilbert space $\mathcal{H}$. The collection $\left\{f_{i j k}\right\}$ satisfies the $U(1)$ cocycle condition $f_{i j k} f_{j k l}^{-1} f_{k l i} f_{l i j}^{-1}=1$ on quadruple overlaps $U_{i} \cap U_{j} \cap U_{k} \cap U_{l}$, and hence they define an integral Cech cocyle

$$
h_{i j k l}=\frac{1}{2 \pi \mathrm{i}}\left(\log f_{i j k}-\log f_{j k l}+\log f_{l i j}\right)
$$

with Čech cohomology class $\left[\left\{h_{i j k l}\right\}\right]=[H] \in H^{3}(\mathcal{B} ; \mathbb{Z})$. In particular, on double overlaps the Hilbert bundles $E_{i}$ and $E_{j}$ differ by a line bundle $L_{i j} \rightarrow U_{i} \cap U_{j}$ with an isomorphism

$$
L_{i j} \otimes L_{j k} \cong L_{i k}
$$

on $U_{i} \cap U_{j} \cap U_{k}$ given by multiplication with $f_{i j k}$. The set of line bundles $\left\{L_{i j}\right\}$ is called a bundle gerbe; the twisted Chan-Paton bundles can then be regarded as bundle gerbe modules associated to $\left\{L_{i j}\right\}$ and the D-brane charges take values in the twisted K-theory defined as the Grothendieck group of the additive category of lifting bundle gerbe modules [35].

In this setting the $B$-field can be described as a connection on a 1-gerbe whose DixmierDouady class is $[H]$, i.e., as a set of two-forms $B_{i}$ on $U_{i}$ satisfying the gluing conditions

$$
B_{j}-B_{i}=\frac{1}{2 \pi \mathrm{i}} \mathrm{d} A_{i j}
$$


on $U_{i} \cap U_{j}$, where $A_{i j}$ is a connection on the line bundle $L_{i j}$ satisfying

$$
A_{i j}+A_{j k}+A_{k i}=\mathrm{d} \log f_{i j k}
$$

on $U_{i} \cap U_{j} \cap U_{k}$; the collection of one-forms $\left\{A_{i j}\right\}$ specifies a set of connections $\nabla_{i}$ on the Hilbert bundles $E_{i} \rightarrow U_{i}$ with the gluing rules

$$
\left.\nabla_{i}\right|_{U_{i} \cap U_{j}}=A_{i j}+\left.\nabla_{j}\right|_{U_{i} \cap U_{j}}
$$

which defines a connection $\nabla$ on the twisted bundle $P_{H} \rightarrow \mathcal{B}$ representing the $B$-field background $(\mathcal{B}, B)$. The $H$-flux is given locally by $\left.H\right|_{U_{i}}=\mathrm{d} B_{i}$ and thus has integral periods on $\mathcal{B}$.

These constructions all carry over to string orbifolds $\mathcal{B}$ by considering bundles on stacks, or equivalently on a presentation of $\mathcal{B}$ by a groupoid $\mathcal{G}=\left(\mathcal{G}_{1} \rightrightarrows \mathcal{G}_{0}\right)$ (cf. Sect. 2). A $B$ field on a groupoid $\mathcal{G}$ is a morphism of groupoids $\tilde{g}: \mathcal{G} \rightarrow P U(\mathcal{H})$ with respect to an open covering $\left\{\mathcal{U}_{i}\right\}$, where here the group $P U(\mathcal{H})$ is regarded as a groupoid $P U(\mathcal{H}) \rightrightarrows\{1\}$. For local quotient groupoids, twisted K-theory is defined in [9] in terms of bundles of Fredholm operators associated with such a twisted bundle of Hilbert spaces.

K-cycles and global anomaly cancellation. The corresponding twisted K-homology cycles $(\mathcal{W}, F, \psi)$ encode the necessary data that must be carried by any D-brane in an $H$-flux background in order to fulfill the Freed-Witten anomaly cancellation conditions [36], such as an $H$-twisted $\operatorname{Spin}^{\mathbb{C}}$ structure and an ordinary complex vector bundle with $[F] \in K^{0}(\mathcal{W})[37$. Cancellation of global worldsheet anomalies in the string theory sigma-model requires

$$
W_{3}(N \mathcal{W})+\psi^{*}[H]=0
$$

in $H^{3}(\mathcal{W} ; \mathbb{Z})$; the integral lift $W_{3}(N \mathcal{W})$ is the obstruction to the existence of a $\operatorname{Spin}^{\mathbb{C}}$ structure on the stabilized normal bundle $N \mathcal{W}$ over the worldvolume $\mathcal{W}$. This is a necessary condition for the homology class $\psi_{*}[\mathcal{W}]$ to lift to twisted K-homology; twisted $\operatorname{Spin}^{\mathbb{C}}$ structures (5.1) are classified topologically by the integral cohomology $H^{2}(\mathcal{W} ; \mathbb{Z})$. To determine if a Dbrane wrapping a non-representable cycle $\mathcal{W}$ carries K-theory charge, one must analyse the worldsheet open string theory, impose boundary conditions corresponding to a singular representative of the cycle, and then check for inconsistencies such as a failure of BRST invariance. Further instabilities can arise if there is a cycle $\psi^{\prime}: \mathcal{W}^{\prime} \rightarrow \mathcal{B}$ such that $\mathcal{W}$ is a codimension three submanifold of $\mathcal{W}^{\prime}$ satisfying the equation [38]

$$
W_{3}\left(N \mathcal{W}^{\prime}\right)+\psi^{*}[H]=\operatorname{Pd}_{\mathcal{W}^{\prime}}(\mathcal{W}),
$$

where $\operatorname{Pd}_{\mathcal{W}^{\prime}}(\mathcal{W})$ is the Poincaré dual class of $[\mathcal{W}]$ in $H^{3}\left(\mathcal{W}^{\prime} ; \mathbb{Z}\right)$.

The original proposal of [39, 40, 41] asserted that D-brane charges in Type IIB string theory in a non-trivial $B$-field background are classified by the twisted K-theory of Rosenberg [42]; in this framework the twisted bundles on the D-brane worldvolumes are bundles of Hilbert spaces associated with the infinite-dimensional locally trivial $C^{*}$-algebra bundle of compact operators $P_{H}(\mathcal{K}):=P_{H} \times_{P U(\mathcal{H})} \mathcal{K}$, where

$$
\mathcal{K}=\underset{n}{\lim } M_{n}(\mathbb{C})
$$


with the limit taken in the $C^{*}$-norm topology on the $n \times n$ matrix algebra $M_{n}(\mathbb{C})$. For our purposes we shall work mostly with the analytic formulation of K-homology as it is this version that is related to the definition of a twisted analogue of K-groups and the Kasparov map, which will allow us to use K-amenability results for Eilenberg-MacLane spaces.

Hyperbolic orbifolds. String backgrounds with hyperbolic orbifold factors become particularly important in the presence of non-vanishing $H$-flux. For example, solutions with brane charges can be found in a conformal field theory for the upper half three-space $H^{3}$ which is constructed as a WZW sigma-model based on the coset $S L(2, \mathbb{C}) / S U(2)$. One can start with a brane solution with flux on $H^{3}$ which is a formal analog of the NS5-brane solution whereby the three-sphere is replaced by a hyperbolic space; its near-horizon geometry describes the background $A d S_{3} \times S^{3} \times H^{3} \times S^{1}$ with a linear dilaton in the time direction [14]. Such a construction leads to a $B$-field with imaginary components, but using S-duality it can be converted into a Ramond-Ramond two-form field with imaginary components which leads to a solution of Type IIB* $^{*}$ supergravity. As a result the conformal sigma-model is an exact solution of Type II string theory to all orders in the $\alpha^{\prime}$-expansion. Using U-duality one can also construct different D-brane solutions with time dependence; in order to accomodate a finite flux, the space $H^{3}$ should be replaced with a finite volume orbifold $H^{3} / \Lambda$.

In a manner akin to our ensuing analysis, a more algebraic perspective on these solutions starts with the observation that the class of Euclidean $A d S_{3}$ spaces we have considered here and in Sect. 2 are quotients of the real hyperbolic space $H^{3}$ by a Schottky group, whose boundaries are compact oriented surfaces with conformal structure. In a similar vein the boundary of the hyperbolic plane $H^{2}$ at infinity is $\mathbb{R} P^{1}$, and its global quotient by a finite index subgroup $\Lambda$ of $G=P S L(2, \mathbb{Z})$ is a modular curve $\mathcal{B}=H^{2} / \Lambda$ which can be presented as the quotient $\mathcal{B}=\left(H^{2} \times G / \Lambda\right) / G$; the degree one homology classes of $\mathcal{B}$ can be regarded as classes in the cyclic cohomology of its noncommutative boundary which is the crossed product $C^{*}$-algebra $C\left(\mathbb{R} P^{1} \times G / \Lambda\right) \rtimes G$ and is Morita equivalent to $C\left(\mathbb{R} P^{1}\right) \rtimes \Lambda$ [43]. More generally, let $X$ be a symmetric space of a real rank one semisimple Lie group, and let $\Lambda \subset G$ be a discrete torsion-free subgroup; then the geodesic boundary of $X$ has a $\Lambda$-equivariant decomposition $\partial X=\Omega_{\Lambda} \cup \Lambda_{\infty}$ where $\Lambda_{\infty}$ is the limit set of $\Lambda$, while the geometric boundary of the Poincaré half-space $H^{n}$ is $\mathbb{R}_{+}^{n-1}$. If $\Lambda$ is convex cocompact (i.e., $X / \Lambda \cup \Omega_{\Lambda}$ is a compact manifold with boundary) then the orbit space $\mathcal{B}=H^{n} / \Lambda$ can be regarded as the interior of a compact manifold with boundary, the Klein manifold $\mathcal{B} \cup\left(\Omega_{\Lambda} / \Lambda\right)$, so that its geometric boundary at infinity is $\Omega_{\Lambda} / \Lambda$; see [44] for further details.

Kasparov theory. Recall [45] that the reduced topological K-theory of the background $\mathcal{B}$ can be defined as the K-theory $\widetilde{K}^{j}(\mathcal{B}) \cong K_{j}\left(C_{0}(\mathcal{B})\right), j=0,1$ of the commutative $C^{*}$-algebra $C_{0}(\mathcal{B})$ of continuous complex-valued functions which vanish at infinity on $\mathcal{B}$. The definition of K-homology involves classifying extensions of $C_{0}(\mathcal{B})$ by the algebra of compact operators $\mathcal{K}$ up to unitary equivalence [46]. The set of homotopy classes of operators defines the Khomology group $K_{0}(\mathcal{B})$, and the duality with K-theory is provided by the natural bilinear index pairing $([\mathcal{E}],[\mathfrak{D}]) \mapsto \operatorname{Index} \mathfrak{D}_{\mathcal{E}} \in \mathbb{Z}$, where $[\mathcal{E}] \in K^{0}(\mathcal{B})$ and $\mathfrak{D}_{\mathcal{E}}$ denotes the action of the Fredholm operator $\mathfrak{D}$ on the Hilbert space $\mathcal{H}=L^{2}(\mathcal{B}, \mathcal{E})$ of square-integrable sections of the vector bundle $\mathcal{E} \rightarrow \mathcal{B}$. If $\mathcal{B}$ is a $\operatorname{Spin}^{\mathbb{C}}$ manifold then there is a Poincaré duality 
isomorphism between compactly supported K-theory and K-homology groups [47].

In this context the KK-pairing appears to be the most natural framework. The group $K K_{\sharp}(A, B)$ is a bivariant version of $\mathrm{K}$-theory which depends on a pair of algebras $A$ and $B$; it defines a homotopy invariant bifunctor from the category of separable $C^{*}$-algebras to the category of abelian groups which depends contravariantly on the algebra $A$ and covariantly on the algebra $B$. It interpolates between K-theory and K-homology in the sense that $K K^{\sharp}(A=\mathbb{C}, B)=K_{\sharp}(B)$ is the K-theory of $B$ while $K K^{\sharp}(A, B=\mathbb{C})=K^{\sharp}(A)$ is the Khomology of $A$. Kasparov's analytic K-homology $K K^{\sharp}\left(C_{0}(\mathcal{B}), \mathbb{C}\right)$ is generated by unitary equivalence classes of (graded) Fredholm modules over $C_{0}(\mathcal{B})$ modulo an operator homotopy relation. See [48, 49] for a description of Kasparov's pairing $\otimes_{D}: K K(A, D) \times K K(D, B) \rightarrow$ $K K(A, B)$ and its properties. Applications of KK-theory to the classification of D-branes can be found in e.g. [50, 51, 36].

In the presence of a non-trivial $B$-field, the K-homology can be defined analogously via the KK-groups of the noncommutative $C^{*}$-algebra $C_{0}\left(\mathcal{B}, P_{H}(\mathcal{K})\right)$ of sections vanishing at infinity of the associated bundle of compact operators $P_{H}(\mathcal{K})$. By identifying the Cech homology of $\mathcal{B}$ with its singular homology, this is the unique (up to isomorphism) stable separable complex continuous trace $C^{*}$-algebra with spectrum $\mathcal{B}$ and Dixmier-Douady class $[H] \in H^{3}(\mathcal{B} ; \mathbb{Z})[42,34$.

For a stack $\mathcal{B}$ with groupoid presentation $\mathcal{G}=\left(\mathcal{G}_{1} \rightrightarrows \mathcal{G}_{0}\right)$, it is convenient to work with a more algebraic definition [52]. For this, we pullback the central extension of groups

$$
1 \longrightarrow U(1) \longrightarrow U(\mathcal{H}) \longrightarrow P U(\mathcal{H}) \longrightarrow 1
$$

by the $B$-field $\tilde{g}: \mathcal{G} \rightarrow P U(\mathcal{H})$ to get a central extension of the groupoid $\mathcal{G}$ which is a principal $U(1)$-bundle $\tilde{\mathcal{G}}_{H} \rightarrow \mathcal{G}$ of groupoids with the same objects $\mathcal{G}_{0}$; for a global quotient stack $[X / \Lambda]$ the gerbe is defined by a central extension $U(1) \rightarrow \tilde{\Lambda}_{H} \stackrel{\mathrm{pr}}{\rightarrow} \Lambda$ and the projection pr induces a $\operatorname{map}\left[X / \tilde{\Lambda}_{H}\right] \rightarrow[X / \Lambda]$. Upon choosing a suitable Haar system on the groupoid, the twisted K-theory can be equivalently computed as the algebraic K-theory of the twisted groupoid $C^{*}$-algebra of $\mathcal{G}$ defined as the subalgebra of $U(1)$-invariants of the convolution groupoid $C^{*}$-algebra $C^{*}\left(\tilde{\mathcal{G}}_{H}\right)\left[52\right.$. Equivalently, as the gerbe associates a multiplier $\zeta \in H^{2}\left(\mathcal{G}_{1} ; U(1)\right)$ on composable arrows, this algebra can be explicitly described as the twisted convolution $C^{*}$-algebra $C^{*}(\mathcal{G}, \zeta)$ of the groupoid $\mathcal{G}$; the two-cocycle $\zeta: \mathcal{G}_{2} \rightarrow U(1)$ represents a class in $H^{2}(\mathcal{B} ; U(1)) \cong H^{3}(\mathcal{B} ; \mathbb{Z})$ and we denote the corresponding $H$-flux by $\left[H_{\zeta}\right]$. A suitable variant of KK-theory in this setting is also defined by [52].

K-amenable groups. Let $A, B$ be unital algebras for which there are elements $\alpha \in$ $K K(A \otimes B, \mathbb{C}), \beta \in K K(\mathbb{C}, A \otimes B)$ with the property $\beta \otimes_{A} \alpha=1_{B} \in K K(B, B), \alpha \otimes_{B} \beta=$ $1_{A} \in K K(A, A)$. Then the algebras $A, B$ have canonically isomorphic $\mathrm{K}$-theory and $\mathrm{K}$ homology, and are said to be KK-equivalent [51]. The notion of KK-equivalence can be used to introduce the concept of K-amenable groups. As before, let $G$ be a connected Lie group and $\Gamma$ a maximal compact subgroup; we further assume that the symmetric space $X=G / \Gamma$ has even dimension and admits a $G$-invariant $\operatorname{Spin}^{\mathbb{C}}$ structure. The $G$-invariant Dirac operator $\mathfrak{D}$ on $X$ is a first order self-adjoint elliptic differential operator acting on $L^{2}$-sections of the $\mathbb{Z}_{2}$-graded homogeneous bundle of spinors $\mathcal{S}$. Consider the zeroth order 
pseudo-differential operator $\mathcal{D}=\mathfrak{D}\left(1+\mathfrak{D}^{2}\right)^{-1 / 2}$ acting on $\mathcal{H}=L^{2}(X, \mathcal{S})$. The algebra $C_{0}(X)$ acts on $\mathcal{H}$ by multiplication operators. The group $G$ acts on $C_{0}(X)$ and $\mathcal{H}$ by left translation, and $\mathcal{D}$ is $G$-invariant. Then the Fredholm module $(\mathcal{D}, \mathcal{H})$ over $C_{0}(X)$ defines a canonical Dirac element $\alpha_{G} \in K K_{G}\left(C_{0}(X), \mathbb{C}\right)$, where $K K_{G}(A, B):=K K(A \rtimes G, B \rtimes G)$ for algebras $A$ and $B$ which admit an action of $G$ by automorphisms. Kasparov shows [48] that there is a canonical Mishchenko element $\beta_{G} \in K K_{G}\left(\mathbb{C}, C_{0}(X)\right)$ with the intersection products

$$
\alpha_{G} \otimes_{\mathbb{C}} \beta_{G}=1_{C_{0}(X)} \in K K_{G}\left(C_{0}(X), C_{0}(X)\right)
$$

and

$$
\beta_{G} \otimes_{C_{0}(X)} \alpha_{G}=\gamma_{G} \in K K_{G}(\mathbb{C}, \mathbb{C})
$$

where $\gamma_{G}$ is an idempotent element in $K K_{G}(\mathbb{C}, \mathbb{C})$. For a semisimple Lie group $G$ or for $G=\mathbb{R}^{n}$, a construction of the Mishchenko element $\beta_{G}$ can be found in [53]. If the group $G$ is amenable, then $\gamma_{G}=1$. All solvable groups are amenable, while any non-compact semisimple Lie group is non-amenable. We therefore say that a Lie group $G$ is K-amenable if $\gamma_{G}=1$.

Proposition 5.3 (a) Any amenable Lie group is K-amenable, and in particular any solvable Lie group is K-amenable.

(b) The non-amenable groups $S O_{0}(n, 1)$ and $S U(n, 1)$ are K-amenable Lie groups [54, 55].

(c) The class of K-amenable groups is closed under the operations of taking subgroups, and of direct and semidirect products [56].

Example: K-amenability in four dimensions. The problem of classifying geometries is one of the central problems in mathematics which also plays a fundamental role in constructing physical models. Every one-dimensional manifold is either $S^{1}$ (closed) or $\mathbb{R}$ (open), with a unique topological piecewise linear smooth structure and orientation. All complex curves of genus zero can be uniformized by rational functions, all those of genus one can be uniformized by elliptic functions, and all those of genus larger than one can be uniformized by meromorphic functions defined on proper open subsets of $\mathbb{C}$. A complete solution to the uniformization problem in higher dimensions has not yet been achieved. In three dimensions, the famous list of Thurston's eight locally homogeneous spaces [57] can be organized into compact stabilizer subgroups $\Gamma_{b}$ of $b \in \mathcal{B}$ which are isomorphic to either $S O(3), S O(2)$ or the trivial group; in the locally symmetric cases the isometry groups are all K-amenable 53. The analogous list of four-geometries (with connected isometry groups) can also be organized as in Tab. 1 [58, 49]. Here we have the four irreducible four-dimensional Riemannian symmetric spaces: sphere $S^{4}$, hyperbolic space $H^{4}$, complex projective space $\mathbb{C} P^{2}$ and complex hyperbolic space $\mathbb{C} H^{2}$, the nilpotent and solvable Lie groups (including $\left.S o l_{m, m}^{4}=S o l^{3} \times \mathbb{R}\right)$, and the space $\mathbb{F}^{4}$ with isometry group $\mathbb{R}^{2} \rtimes P S L(2, \mathbb{R})$.

Corollary 5.4 The isometry groups $G$ of the locally symmetric backgrounds $\mathcal{B}=\Lambda \backslash X$ listed in Tab. 1 are all K-amenable. 
Table 1. Four-geometries

Stabilizer subgroup $\Gamma \quad$ Background $\mathcal{B}=\Lambda \backslash G / \Gamma$

$\begin{array}{ll}S O(4) & S^{4}, \mathbb{R}^{4}, H^{4} \\ U(2) & \mathbb{C} P^{2}, \mathbb{C} H^{2} \\ S O(2) \times S O(2) & S^{2} \times \mathbb{R}^{2}, S^{2} \times S^{2}, S^{2} \times H^{2}, H^{2} \times \mathbb{R}^{2}, H^{2} \times H^{2} \\ S O(3) & S^{3} \times \mathbb{R}, H^{3} \times \mathbb{R} \\ S O(2) & N i l^{3} \times \mathbb{R}, \widetilde{P S L}(2, \mathbb{R}) \times \mathbb{R}, S_{S o l}^{4} \\ U(1) & \mathbb{F}^{4} \\ \text { trivial } & N i l^{4}, \text { Sol }_{m, n}^{4}, S o l_{1}^{4}\end{array}$

To establish this result we need to prove that $\gamma_{G}=1$ for each of the four-geometries occuring in Tab. 1. Let us consider just a few representative examples, see 49 for further details:

- $G=\mathbb{R}^{4} \rtimes S O(4), X=\mathbb{R}^{4}$ : By Prop. 5.3, $\gamma_{G}=1$ since $\mathbb{R}^{4}$ and $S O(4)$ are amenable, and so is their semidirect product.

- $G=S O_{0}(4,1), X=H^{4}: \gamma_{G}=1$ by Prop. 5.3.

$-G=S U(3), X=\mathbb{C} P^{2} \cong U(3) /(U(1) \times U(2)) \cong S U(3) / S(U(1) \times U(2)): \gamma_{G}=1$ by Prop. 5.3.

- $X=\mathbb{C} H^{2}$ : The four-geometry $\mathbb{C} H^{2}$ is a Kählerian symmetric space and carries a complex structure, hence $\gamma_{G}=1$.

- $X=H^{2} \times \mathbb{R}^{2}, H^{2} \times H^{2}, H^{3} \times \mathbb{R}$ : By Prop. 5.3 one has $\gamma_{G}=1$ since these isometry groups are direct products of $\mathrm{K}$-amenable groups.

Twisted K-groups. Let $A$ be an algebra admitting an action of a lattice $\Lambda \subset G$ by automorphisms. The crossed product algebra $\left(A \otimes C_{0}(X)\right) \rtimes \Lambda$ is Morita equivalent to the algebra $C_{0}(\mathcal{B}, \mathcal{E})$ of continuous sections vanishing at infinity of the flat $A$-bundle defined by the quotient $\mathcal{E}:=\Lambda \backslash(X \times A) \rightarrow \mathcal{B}$ with the diagonal action of $\Lambda$ on $X \times A$ [53]. One has the following version of the Thom isomorphism theorem for the K-theory of $C^{*}$-algebras.

Theorem 5.5 [48] If $G$ is K-amenable, then $(A \rtimes \Lambda) \otimes C_{0}(X)$ and $\left(A \otimes C_{0}(X)\right) \rtimes \Lambda$ have the same K-theory.

It follows that when $G$ is $\mathrm{K}$-amenable, the algebras $(A \rtimes \Lambda) \otimes C_{0}(X)$ and $C_{0}(\mathcal{B}, \mathcal{E})$ have the same K-theory so that

$$
K_{\sharp}\left(C_{0}(\mathcal{B} ; \mathcal{E})\right) \cong K_{\sharp+\operatorname{dim}(X)}(A \rtimes \Lambda) .
$$


Let $\zeta \in H^{2}(\Lambda ; U(1))$ be a multiplier on $\Lambda$, i.e., a normalized $U(1)$-valued group two-cocycle on $\Lambda$.

Theorem 5.6 [53] If $G$ is K-amenable, then

$$
K_{\sharp}\left(C^{*}(\Lambda, \zeta)\right) \cong K^{\sharp+\operatorname{dim}(X)}\left(\mathcal{B},\left[H_{\zeta}\right]\right),
$$

where $K^{\sharp}\left(\mathcal{B},\left[H_{\zeta}\right]\right)$ is the twisted $K$-theory of the continuous trace $C^{*}$-algebra $C_{0}\left(\mathcal{B}, P_{H_{\zeta}}(\mathcal{K})\right)$ with Dixmier-Douady invariant $\left[H_{\zeta}\right] \in H^{3}(\mathcal{B} ; \mathbb{Z})$.

To better understand this result, first suppose that $A=\mathbb{C}$ with $\Lambda$ acting trivially. When $\gamma_{G}=1$, Thm. 5.5 implies that the algebras $(\mathbb{C} \rtimes \Lambda) \otimes C_{0}(X)$ and $C_{0}(\mathcal{B}, \mathcal{E})$ have the same K-theory, where $\mathcal{E}$ is the trivial complex line bundle over $\mathcal{B}$; it follows that $K_{\sharp}\left(C^{*}(\Lambda)\right) \cong$ $K^{\sharp+\operatorname{dim}(X)}(\mathcal{B})$. Now suppose that $\zeta \in H^{2}(\Lambda ; U(1))$. If $G$ is $\mathrm{K}$-amenable, then Thm. 5.5 and the Packer-Raeburn stabilization trick [59] imply that $\left(A \rtimes_{\zeta} \Lambda\right) \otimes C_{0}(X)$ and $C_{0}\left(\mathcal{B}, \mathcal{E}_{\zeta}\right)$ have the same K-theory, where $\mathcal{E}_{\zeta}:=\Lambda \backslash(X \times(A \otimes \mathcal{K})) / \Lambda \rightarrow \mathcal{B}$ with $\mathcal{K}$ the algebra of compact operators. Since by definition the twisted K-theory $K^{\sharp}\left(\mathcal{B},\left[H_{\zeta}\right]\right)$ is the K-theory of the continuous trace $C^{*}$-algebra $C_{0}\left(\mathcal{B}, \mathcal{E}_{\zeta}\right)$, Eq. (5.7) thus follows. We may regard this result as saying that twisted D-brane charges in the $H$-flux background are the same as those of D-branes in a noncommutative deformation of the locally symmetric space $\mathcal{B}$ induced by a generating $B$-field.

Examples. (i) General theorems regarding the K-groups of $C^{*}$-algebras are obtained in [60, 61, 62], while the analysis of Baum-Connes type conjectures concerning the K-theories of twisted group $C^{*}$-algebras is carried out in [63, 64]. Collecting these results in the generic case one finds $K_{\sharp}\left(C^{*}\left(\mathbb{Z}^{n}, \zeta\right)\right) \cong K_{\sharp}\left(C^{*}\left(\mathbb{Z}^{n}\right)\right) \cong K^{\sharp}\left(T^{n}\right)$ for any multiplier $\zeta$ on $\mathbb{Z}^{n}$. The twisted group $C^{*}$-algebras $C^{*}\left(\mathbb{Z}^{n}, \zeta\right)$ are the well-known noncommutative $n$-tori.

(ii) When $\Lambda=\Lambda_{g}$ is the fundamental group of a Riemann surface $\Sigma_{g}$ of genus $g>$ 0 , the Dixmier-Douady class $\left[H_{\zeta}\right]$ is trivial and we get [53] $K_{0}\left(C^{*}\left(\Lambda_{g}, \zeta\right)\right) \cong K^{0}\left(\Sigma_{g}\right) \cong$ $\mathbb{Z}^{2}, K_{1}\left(C^{*}\left(\Lambda_{g}, \zeta\right)\right) \cong K^{1}\left(\Sigma_{g}\right) \cong \mathbb{Z}^{2 g}$ for any multiplier $\zeta$ on $\Lambda_{g}$. The twisted group $C^{*}$ algebra $C^{*}\left(\Lambda_{g}, \zeta\right)$ is called a noncommutative Riemann surface.

(iii) Since the symmetric space $X=G / \Gamma$ is contractible and the group $\Lambda$ acts freely on $X$, one has $H_{i}(\Lambda ; A)=H_{i}(\mathcal{B} ; A)$ and $H^{i}(\Lambda ; A)=H^{i}(\mathcal{B} ; A)$ for any trivial $\Lambda$-module $A$ where the groups on the left-hand sides are Eilenberg-MacLane homology and cohomology groups. As an explicit example, let $G=\mathbb{R}^{n}, \Lambda=\mathbb{Z}^{n}$, and $\Gamma=\{0\}$. In this case $\mathcal{B}=\Lambda \backslash G / \Gamma=T^{n}$ is an $n$-torus with

$$
H_{i}\left(T^{n} ; \mathbb{Z}\right)=H^{i}\left(T^{n} ; \mathbb{Z}\right)=H^{i}\left(\mathbb{Z}^{n} ; \mathbb{Z}\right)=\mathbb{Z}^{\left(\begin{array}{l}
n \\
i
\end{array}\right)}
$$

Then for $j=0,1$ one has

$$
K_{j}\left(T^{n}\right)=K^{j}\left(T^{n}\right)=\bigoplus_{i=0}^{\left\lfloor\frac{n}{2}\right\rfloor} H^{2 i+j}\left(T^{n} ; \mathbb{Z}\right)=\prod_{i=0}^{\left\lfloor\frac{n}{2}\right\rfloor} \mathbb{Z}^{\left(\begin{array}{c}
n \\
2 i+j
\end{array}\right)}=\mathbb{Z}^{2^{n-1}}
$$

The power $2^{n-1}$ gives the expected multiplicity of brane charges arising from wrapping all higher stable D-branes on various cycles of the torus $T^{n}$ [65]. 
Eilenberg-MacLane spectrum functor. One of the main results of [53] says that for lattices in $\mathrm{K}$-amenable Lie groups the reduced and unreduced twisted group $C^{*}$-algebras have canonically isomorphic K-theory. If $\zeta \in H^{2}(\Lambda, U(1))$ is a multiplier on a lattice $\Lambda$ in a K-amenable Lie group $G$, then the canonical morphism $C^{*}(\Lambda, \zeta) \rightarrow C_{r}^{*}(\Lambda, \zeta)$ induces an isomorphism

$$
K_{\sharp}\left(C^{*}(\Lambda, \zeta)\right) \cong K_{\sharp}\left(C_{r}^{*}(\Lambda, \zeta)\right) .
$$

If moreover $\operatorname{dim}(X)=3$ and $\Lambda$ is a uniform lattice in $G$, then for $j=0,1$ one has

$$
K_{j}\left(C_{r}^{*}(\Lambda, \zeta)\right) \cong K_{j}\left(C_{r}^{*}(\Lambda)\right) \cong K^{j+1}(\mathcal{B})
$$

To show this, we use Thm. 5.6 to get $K_{j}\left(C_{r}^{*}(\Lambda)\right) \cong K^{j+\operatorname{dim}(X)}(\mathcal{B})$ for $j=0,1$. By the Packer-Raeburn stabilization trick [59], $C_{r}^{*}(\Lambda, \zeta)$ is Morita equivalent to $\mathcal{K} \rtimes \Lambda$ and since $G$ is $\mathrm{K}$-amenable $(\mathcal{K} \rtimes \Lambda) \otimes C_{0}(X)$ is Morita equivalent to $C_{0}\left(\mathcal{B}, \mathcal{E}_{\zeta}\right)$, where as before $\mathcal{E}_{\zeta}$ is a locally trivial bundle of $C^{*}$-algebras over $\mathcal{B}$ with fiber $\mathcal{K}$. The Dixmier-Douady invariant is an element $\left[H_{\zeta}\right] \in H^{3}(\mathcal{B} ; \mathbb{Z}) \cong H^{3}(\Lambda ; \mathbb{Z})$. If $\mathcal{B}$ is not orientable, then $H^{3}(\mathcal{B} ; \mathbb{Z})=\{0\}$; therefore $\left[H_{\zeta}\right]=0$ and Eq. (5.8) holds. On the other hand, when $\mathcal{B}$ is orientable one has $H^{3}(\mathcal{B} ; \mathbb{Z}) \cong \mathbb{Z}$ but $\left[H_{\zeta}\right]=0$ for all $\zeta \in H^{2}(\Lambda, U(1))\left[53\right.$, and so $C_{0}\left(\mathcal{B}, \mathcal{E}_{\zeta}\right)$ is Morita equivalent to $C_{0}(\mathcal{B})$; in this case we have again Eq. (5.8).

Let $\underline{K}(\Lambda, n)$ be an Eilenberg-MacLane space, i.e., the unique space (up to homotopy) whose homotopy groups are $\pi_{m}(\underline{K}(\Lambda, n))=0$ for all $m \neq n$ and $\pi_{n}(\underline{K}(\Lambda, n))=\Lambda$; for example one has $\underline{K}\left(\mathbb{Z}^{n}, 1\right)=T^{n}$ and $\underline{K}(\mathbb{Z}, 2)=\mathbb{C} P^{\infty} \cong \underline{B} U(1)$ is the classifying space of $U(1)$-bundles, while $\underline{K}(\mathbb{Z}, 2)=P U(\mathcal{H})$ and $\underline{K}(\mathbb{Z}, 3)=\underline{B} P U(\mathcal{H})$ is the classifying space of twisted bundles. The spaces $\{\underline{K}(\Lambda, n)\}_{n \geq 0}$ fit together via the loop space functor to form the EilenbergMacLane spectrum

$$
\cdots \stackrel{\Omega}{\longleftarrow} \underline{K}(\Lambda, n) \stackrel{\Omega}{K} \underline{K}(\Lambda, n+1) \stackrel{\Omega}{\longleftarrow} \cdots
$$

which we can regard as a functor from the category of abelian groups to the category of loop spectra.

Any compact connected locally symmetric background $\mathcal{B}$ is an Eilenberg-MacLane space $\underline{K}(\Lambda, 1)$. Conversely, if $\underline{K}(\Lambda, 1)$ is a locally symmetric manifold then it is of the form $\Lambda \backslash G / \Gamma$ where $G$ is a connected Lie group, $\Gamma$ is a maximal compact subgroup of $G$ and $\Lambda$ is a uniform lattice in $G$. In [53] it is conjectured that Eq. (5.8) is still valid without the assumption of local symmetry: If $\mathcal{B}$ is a connected compact three-manifold which is an Eilenberg-MacLane space with fundamental group $\Lambda$, then for any multiplier $\zeta \in H^{2}(\Lambda, U(1))$ on $\Lambda$ one has isomorphisms $K_{j}\left(C_{r}^{*}(\Lambda, \zeta)\right) \cong K_{j}\left(C_{r}^{*}(\Lambda)\right) \cong K^{j+1}(\mathcal{B})$ for $j=0,1$. This statement is extended to the four-manifolds of Tab. 1 in [49].

Brane stability conditions. The problem of stability of the supergravity solutions discussed above can be embedded into the framework of Sect. 4. Given a stratified system of fiber bundles $p: X \rightarrow \mathcal{B}$ over a hyperbolic orbifold which supports a non-trivial $B$-field $P_{H} \rightarrow \mathcal{B}$, one can repeat the topological classification of stable branes in terms of Quinn homology groups $Q_{i}(\mathcal{B} ; \mathcal{G}(p))$ by using spectra suitable to the appropriate twisted homology theory; in this case we assume that $\mathcal{B}$ can be covered by suitable neighborhoods $U_{i}$ of the 
strata $\mathcal{B}_{i}$. For D-branes, we identify the Bott spectrum $\mathcal{K}$ with the iterated loop spectrum of the space of Fredholm operators and choose a spectrum-valued functor of spaces $\mathcal{G}$ such that $\mathcal{G}(X)$ is homotopy equivalent to the loop spectrum which is the pullback by $p$ of the associated bundle of K-homology spectra $P_{H}(\mathcal{K}):=P_{H} \times_{P U(\mathcal{H})} \mathcal{K}$. In this case anomaly cancellation (5.1) and instability (5.2) are consistent as before with the homology of the differential $\mathrm{d}_{i, 0}^{3}$ in the spectral sequence extension problem [38], but unfortunately not very much is known about the higher differentials in general. Other branes can be obtained by duality.

A somewhat simpler analysis can be carried out using Eilenberg-MacLane spaces. If $A$ is a stratified system of abelian groups over $\mathcal{B} \supset \mathcal{B}^{i} \supset \mathcal{B}^{i-1} \supset \cdots$ as in Sect. 4 , we can apply the Eilenberg-MacLane spectrum functor to its fibers. For each $b \in \mathcal{B}$ with $b \in \mathcal{B}^{i}$ this gives fiber bundles

$$
\underline{K}(A(b), n) \longrightarrow \underline{K}\left(A_{i}, n\right) \longrightarrow U_{i} .
$$

The homomorphisms $\theta_{i j}: A_{i} \rightarrow A_{j}$ define fiber maps over $U_{i} \cap U_{j}$ which can be used to define a topology on the disjoint union $\underline{K}(A, n):=\coprod_{i \geq 1} \underline{K}\left(A_{i}, n\right)$. Applying the EilenbergSteenrod additivity axiom to any sequence of homology functors $H_{n}$ gives

$$
H_{\sharp}(\underline{K}(A, n)) \cong \bigoplus_{i \geq 1} H_{\sharp}\left(\underline{K}\left(A_{i}, n\right)\right) .
$$

If each $\mathcal{B}^{i}$ is an absolute neighborhood retract (i.e., $\mathcal{B}^{i}$ has a neighborhood which retracts it when embedded as a closed subspace of a normal space), then $\underline{K}(A, n) \rightarrow \mathcal{B}$ is a stratified system of fiber bundles which fit together to give a spectral sheaf $\mathcal{G}_{A}$ over $\mathcal{B}$. The corresponding homology spectrum is denoted $\mathcal{Q}(\mathcal{B} ; A):=\mathcal{Q}\left(\mathcal{B} ; \mathcal{G}_{A}\right)$, and as before the Quinn homology groups are computed from the homotopy of this spectrum as $Q_{j}(\mathcal{B} ; A)=\pi_{j} \mathcal{Q}(\mathcal{B} ; A)$.

By Sect. 3 these homology groups may be computed from an Atiyah-Hirzebruch type spectral sequence with $E^{2}$ term

$$
E_{i, j}^{2} \cong \bigoplus_{k \geq 1} H_{i}\left(\mathcal{B} ; \pi_{j}\left(\mathcal{G}_{A_{k}}\right)\right)
$$

As in Sect. 4, if $\underline{K}(A, n)$ extends through this spectral sequence as a non-trivial element of all homology groups, then it can pass to a non-trivial element of $E_{i, j}^{\infty}$ and hence have a non-trivial lift to K-homology. Then the twisted additive structure of the K-homology cycles will encode the lower brane charges that must be carried by any D-brane which wraps the Eilenberg-MacLane space $\underline{K}(A, n)$.

This construction provides a novel mechanism for stability of the hyperbolic orbifold background $\mathcal{B}$, wherein it can be extended via a stratified system of groups to a non-trivial element of K-homology. As the homotopy groups of $\underline{K}(A, n)$ are mostly trivial, this imposes much more stringent constraints on the allowed stable branes in such $B$-field backgrounds. A model for each space $\underline{K}\left(A_{i}, n\right)$ can be obtained by taking a presentation of $A_{i}$ and the smash product $S^{n} \wedge \cdots \wedge S^{n}$, with one $n$-sphere for each generator of $A_{i}$, and then attaching $n+1$ cells for each relation. If $n=1$ and $A_{i}$ has torsion, then no finite-dimensional CW-complex can model $\underline{K}\left(A_{i}, 1\right)$ as then its homology is torsion in each odd degree by the Dold-Thom theorem; for example $\underline{K}\left(\mathbb{Z}_{m}, 1\right)$ can be modelled by the infinite Lens space $S^{\infty} / \mathbb{Z}_{m}$. 


\section{Acknowledgments}

We are grateful to Alan Carey and Masud Chaichian for helpful discussions and suggestions. AAB would like to acknowledge the Conselho Nacional de Desenvolvimento Científico e Tecnológico (CNPq, Brazil) and Fundaçao Araucaria (Parana, Brazil) for financial support. The support of the Academy of Finland under the Projects No. 136539 and 272919 is gratefully acknowledged. The work of RJS was partially supported by the Consolidated Grant ST/L000334/1 from the UK Science and Technology Facilities Council. Part of this work was carried out while RJS was visiting the Hausdorff Research Institute for Mathematics in Bonn during the 2014 Trimester Program "Noncommutative Geometry and its Applications"; he would like to thank Alan Carey, Victor Gayral, Matthias Lesch, Walter van Suijlekom and Raimar Wulkenhaar for the invitation, and all the staff at HIM for the warm hospitality.

\section{References}

[1] L. J. Dixon, J. A. Harvey, C. Vafa and E. Witten, Strings on Orbifolds, Nucl. Phys. B 261 (1985) 678-686.

[2] E. R. Sharpe, String Orbifolds and Quotient Stacks, Nucl. Phys. B 627 (2002) 445-505; arXiv:hep-th/0102211.

[3] T. Pantev and E. R. Sharpe, String Compactifications on Calabi-Yau Stacks, Nucl. Phys. B 733 (2006) 233-296; arXiv:hep-th/0502044.

[4] J. Figueroa-O'Farrill and N. Hustler, The Homogeneity Theorem for Supergravity Backgrounds, JHEP 1210 (2012) 014; [arXiv:hep-th/1208.0553].

[5] F. Quinn, Ends of Maps II, Invent. Math. 68 (1982) 353-424.

[6] A. Adem, J. Leida and Y. Ruan, Orbifolds and Stringy Topology (Cambridge University Press, Cambridge, 2007).

[7] J. Heinloth, Notes on Differentiable Stacks, in: Mathematisches Institut Georg-AugustUniversität Göttingen Seminars Winter Term 2004/2005, ed. Yu. Tschinkel (Universitätsdrucke Göttingen, 2005) 1-32.

[8] B. Hughes, Stratified Path Spaces and Fibrations, Proc. Roy. Soc. Edinburgh A 129 (1999) 351-384.

[9] D. S. Freed, M. J. Hopkins and C. Teleman, Loop Groups and Twisted K-Theory I, J. Topol. 4 (2011) 737-798; [arXiv:math.AT/0711.1906].

[10] P. Breitenlohner and D. Z. Freedman, Stability in Gauged Extended Supergravity, Ann. Phys. 144 (1982) 249-281. 
[11] Y. Fujii and K. Yamagishi, Killing Spinors on Spheres and Hyperbolic Manifolds, J. Math. Phys. 27 (1986) 979-981.

[12] H. Lu, C. N. Pope and P. K. Townsend, Domain Walls from Anti-de Sitter Spacetime, Phys. Lett. B 391 (1997) 39-46; arXiv:hep-th/9607164.

[13] H. Lu, C. N. Pope and J. Rahmfeld, A Construction of Killing Spinors on $S^{n}$, J. Math. Phys. 40 (1999) 4518-4526; arXiv:hep-th/9805151].

[14] A. Kehagias and J. G. Russo, Hyperbolic Spaces in String and M-Theory, JHEP 0007 (2000) 027; arXiv:hep-th/0003281.

[15] S. Kachru and E. Silverstein, 4d Conformal Field Theories and Strings on Orbifolds, Phys. Rev. Lett. 80 (1998) 4855-4858; arXiv:hep-th/9802183.

[16] A. Lawrence, N. A. Nekrasov and C. Vafa, On Conformal Theories in Four Dimensions, Nucl. Phys. B 533 (1998) 199-209; |arXiv:hep-th/9803015].

[17] T. Friedrich, Dirac Operators in Riemannian Geometry, Grad. Stud. Math. 25 (1997) $1-193$.

[18] R. N. Talbert, An Isomorphism between Bredon and Quinn Homology, Forum Math. 11 (1999) 591-616.

[19] A. K. Bousfield and D. M. Kan, Homotopy Limits, Completions and Localizations, Lect. Notes Math. 304 (1972) 1-348.

[20] G. W. Whitehead, Generalized Homology Theories, Trans. Amer. Math. Soc. 102 (1962) $227-283$.

[21] I. M. James, Ex-Homotopy Theory, Illinois J. Math. 15 (1971) 324-337.

[22] G. W. Whitehead, Elements of Homotopy Theory, Grad. Texts Math. 61 (1988) 1-736.

[23] F. T. Farrell and L. E. Jones, Algebraic K-Theory of Spaces Stratified Fibered over Hyperbolic Orbifolds, Proc. Natl. Acad. Sci. USA 83 (1986) 5364-5366.

[24] J. F. Davis and W. Lück, Spaces over a Category and Assembly Maps in Isomorphism Conjectures in K- and L-Theory, K-Theory 15 (1998) 201-252.

[25] R. J. Szabo and A. Valentino, Ramond-Ramond Fields, Fractional Branes and Orbifold Differential K-Theory, Commun. Math. Phys. 294 (2010) 647-702; [arXiv:hepth/0710.2773].

[26] A. A. Bytsenko, M. Chaichian and M. E. X. Guimarães, D-Branes on Spaces Stratified Fibered Over Hyperbolic Orbifolds, Int. J. Mod. Phys. A 29 (2014) 1450137; [arXiv:hepth/1408.1071].

[27] R. M. G. Reis and R. J. Szabo, Geometric K-Homology of Flat D-Branes, Commun. Math. Phys. 266 (2006) 71-122; arXiv:hep-th/0507043. 
[28] D.-E. Diaconescu, G. W. Moore and E. Witten, E $E_{8}$ Gauge Theory and a Derivation of K-Theory from M-Theory, Adv. Theor. Math. Phys. 6 (2003) 1031-1134; arXiv:hep-th/0005090.

[29] D. Quillen, Finite Generation of the Groups $K_{i}$ of Rings of Algebraic Integers, Lect. Notes Math. 341 (1973) 179-198.

[30] A. Borel, Stable Real Cohomology of Arithmetic Groups, Ann. Sci. École Norm. Sup. 7 (1974) 235-272.

[31] R. Miatello, On the Plancherel Measure for Linear Lie Groups of Rank One, Manuscr. Math. 29 (1979) 249-276.

[32] R. Miatello, The Minakshisundaram-Pleijel Coefficients for the Vector-Valued Heat Kernel on Compact Locally Symmetric Spaces of Negative Curvature, Trans. Amer. Math. Soc. 260 (1980) 1-33.

[33] A. A. Bytsenko and F. L. Williams, Asymptotics of the Heat Kernel on Rank One Locally Symmetric Spaces, J. Phys. A 32 (1999) 5773-5780; arXiv:math.SP/9804115.

[34] M. F. Atiyah and G. B. Segal, Twisted K-Theory, Ukr. Math. Bull. 1 (2004) 291-334; arXiv:math.KT/0510674.

[35] P. Bouwknegt, A. L. Carey, V. Mathai, M. K. Murray and D. Stevenson, Twisted KTheory and K-Theory of Bundle Gerbes, Commun. Math. Phys. 228 (2002) 17-49; arXiv:hep-th/0106194.

[36] R. J. Szabo, D-Branes and Bivariant K-Theory, Keio COE Lect. Ser. Math. Sci. 1 (2013) 131-175; [arXiv:hep-th/0809.3029].

[37] A. L. Carey and B.-L. Wang, Riemann-Roch and Index Formulae in Twisted K-Theory, Proc. Symp. Pure Math. 81 (2010) 95-131; [arXiv:math.KT/0909.4848].

[38] J. M. Maldacena, G. W. Moore and N. Seiberg, D-Brane Instantons and K-Theory Charges, JHEP 0111 (2001) 062; arXiv:hep-th/0108100].

[39] E. Witten, D-Branes and K-Theory, JHEP 9812 (1998) 019; arXiv:hep-th/9810188.

[40] A. Kapustin, D-Branes in a Topologically Nontrivial B-field, Adv. Theor. Math. Phys. 4 (2000) 127-154; arXiv:hep-th/9909089.

[41] P. Bouwknegt and V. Mathai, D-Branes, B-Fields and Twisted K-Theory, JHEP 0003 (2000) 007; arXiv:hep-th/0002023.

[42] J. Rosenberg, Continuous Trace Algebras from the Bundle Theoretic Point of View, J. Austral. Math. Soc. 47 (1989) 368-381.

[43] Yu. I. Manin and M. Marcolli, Holography Principle and Arithmetic of Algebraic Curves, Adv. Theor. Math. Phys. 5 (2002) 617-650; arXiv:hep-th/0201036]. 
[44] A. A. Bytsenko, M. E. X. Guimarães and J. A. Helayel-Neto, Hyperbolic Space Forms and Orbifold Compactification in M-Theory, PoS WC 2004 (2004) 017; arXiv:hep-th/0502031.

[45] N. E. Wegge-Olsen, K-Theory and $C^{*}$-Algebras (Oxford University Press, Oxford, 2000).

[46] L. Brown, R. G. Douglas and P. Fillmore, Extensions of $C^{*}$-Algebras and K-Homology, Ann. Math. 105 (1977) 265-324.

[47] N. Higson and J. Roe, Analytic K-Homology (Oxford University Press, Oxford, 2000).

[48] G. G. Kasparov, K-Theory, Group $C^{*}$-Algebras and Higher Signatures, London Math. Soc. Lect. Notes 226 (1995) 101-146.

[49] A. A. Bytsenko, Homology and K-Theory Methods for Classes of Branes Wrapping Nontrivial Cycles, J. Phys. A 41 (2008) 045402; [arXiv:hep-th/0710.0305].

[50] V. Periwal, D-Brane Charges and K-Homology, JHEP 0007 (2000) 041; arXiv:hep-th/0006223.

[51] J. Brodzki, V. Mathai, J. Rosenberg and R. J. Szabo, D-Branes, RR-Fields and Duality on Noncommutative Manifolds, Commun. Math. Phys. 277 (2008) 643-706; arXiv:hep-th/0607020.

[52] J. L. Tu, P. Xu and C. Laurent, Twisted K-Theory of Differentiable Stacks, Ann. Sci. École Norm. Sup. 37 (2004) 841-910; arXiv:math.KT/0306138.

[53] A. L. Carey, K. C. Hannabuss, V. Mathai and P. McCann, Quantum Hall Effect on the Hyperbolic Plane, Commun. Math. Phys. 190 (1998) 629-673; arXiv:dg-ga/9704006.

[54] G. G. Kasparov, Lorentz Groups, K-Theory of Unitary Representations and Crossed Products, Sov. Math. Dokl. 29 (1984) 256-260.

[55] P. Julg and G. G. Kasparov, Operator K-Theory for the Group $S U(n, 1)$, J. Reine Angew. Math. 463 (1995) 99-152.

[56] J. Cuntz, K-Theoretic Amenability for Discrete Groups, J. Reine Angew. Math. 344 (1983) 180-195.

[57] W. Thurston, Three-Dimensional Manifolds, Kleinian Groups and Hyperbolic Geometry, Bull. Amer. Math. Soc. 6 (1982) 357-381.

[58] B. N. Apanasov, Geometry of Discrete Groups and Manifolds (Nauka, Moscow, 1991).

[59] J. Packer and I. Raeburn, Twisted Crossed Products of $C^{*}$-Algebras, Math. Proc. Cambridge Phil. Soc. 106 (1989) 293-311.

[60] G. Elliott, On the K-Theory of the $C^{*}$-Algebra Generated by a Projective Representation of a Torsion-Free Discrete Group, Monogr. Stud. Math. 17 (1984) 157-184. 
[61] J. Bellissard, K-Theory of $C^{*}$-Algebras in Solid State Physics, Lect. Notes Phys. 257 (1986) 99-156.

[62] A. Connes, Noncommutative Differential Geometry, Publ. Math. IHES 62 (1986) 257360 .

[63] M. Marcolli and V. Mathai, Twisted Index Theory on Good Orbifolds I: Noncommutative Bloch Theory, Commun. Contemp. Math. 1 (1999) 553-587; arXiv:math.DG/9911102.

[64] V. Mathai, Heat Kernels and the Range of the Trace on Completions of Twisted Group Algebras, Contemp. Math. 398 (2006) 321-346; arXiv:math.KT/0606790].

[65] K. Olsen and R. J. Szabo, Constructing D-Branes from K-Theory, Adv. Theor. Math. Phys. 3 (1999) 889-1025; arXiv:hep-th/9907140. 